\title{
1 A negative feedback loop of the TOR signaling moderates 2 growth and enables rapid sensing of stress signals in plants
}

3 Muhammed Jamsheer $\mathrm{K}^{1 \#{ }^{*}<}$, Sunita Jindal ${ }^{1 \#>}$, Mohan Sharma ${ }^{1}$, Manvi Sharma ${ }^{1}$, Sreejath Sivaj ${ }^{2}$, 4 Chanchal Thomas Mannully ${ }^{1 \wedge}$, Ashverya Laxmi ${ }^{1 *}$

$5 \quad{ }^{1}$ National Institute of Plant Genome Research, Aruna Asaf Ali Marg, New Delhi 110067, India

6 'Department of Mechanical Engineering, Indian Institute of Technology Delhi, New Delhi, 110016, 7 India

8

$<$ Current affiliation: Amity Food \& Agriculture Foundation, Amity University Uttar Pradesh, Sector 125, Noida 201313, India

^Current affiliation: Cell Metabolism Laboratory, School of Pharmacy, The Hebrew University of Jerusalem, Jerusalem, 9112102, Israel

>Current affiliation: Department of Molecular Biology and Radiology, Mendel University in Brno, Brno, 613 00, Czech Republic

\#Equal first authors

${ }^{*}$ Corresponding authors

19 Ashverya Laxmi

20 Muhammed Jamsheer $\mathrm{K}$

21 Email: AL: ashverya laxmi@nipgr.ac.in (Lead Contact); MJK: mjamsheerk@amity.edu

22 ORCID: Ashverya Laxmi (0000-0002-3430-4200); Muhammed Jamsheer K (0000-0002-2135-

23 8760) 


\section{ABSTRACT}

TOR kinase is a central coordinator of nutrient-dependent growth in eukaryotes. Maintaining optimal TOR signaling is critical for the normal development of organisms. However, the mechanisms involved in the maintenance of optimal TOR signaling are currently unknown in plants. In this study, we describe a negative feedback loop of TOR signaling helping in the adaptability of plants in changing environmental conditions. Using an interdisciplinary approach,

42 we identified a plant-specific zinc finger protein FLZ8, as a regulator of TOR signaling in 43 Arabidopsis. In sugar sufficiency, FLZ8 is upregulated by TOR-dependent and -independent 44 histone modifications. FLZ8 negatively regulates TOR signaling by promoting antagonistic 45 SnRK1a1 signaling and bridging the interaction of SnRK1a1 with RAPTOR, a crucial accessory protein of TOR. This negative feedback loop moderates the TOR-growth signaling axis in the favorable condition and helps in the rapid activation of stress signaling in unfavorable conditions establishing its importance in the adaptability of plants.

49

50 


\section{INTRODUCTION}

71 Organisms need to coordinate their growth according to nutrient availability. The Target Of 72 Rapamycin (TOR) kinase works as a central regulator of growth in nutrient sufficiency ${ }^{1,2}$. Sugar 73 and other nutrients (nitrogen, amino acids, phosphate, etc.) activate TOR complex 1 (TORC1), 74 which is composed of TOR and the accessory proteins, Regulatory-associated protein of TOR 75 (RAPTOR) and Lethal with Sec Thirteen 8 (LST8) ${ }^{1}$. TORC1 promotes general anabolism and 76 growth ${ }^{2}$. Nutrient starvation especially the sugar starvation, activates AMP-activated protein kinase (AMPK) which reprograms the growth according to the available nutrients ${ }^{3}$. The plant homolog of AMPK is known as Snf1-related protein kinase 1 (SnRK1) is found to be a master regulator of growth in sugar starvation indicating the functional conservation of this pathway ${ }^{4}$. AMPK/SnRK1 phosphorylates RAPTOR leading to the suppression of TOR during starvation 5,6 . Recently, TORC1 was found to suppress AMPK activity through the direct phosphorylation of its $\alpha$ kinase subunit during starvation ${ }^{7}$. Thus, the double-negative feedback loop between TOR and AMPK/SnRK1 coordinates growth in eukaryotes according to nutrient availability ${ }^{1}$.

$T O R$ is an essential gene and disruption of TOR causes lethal defects in embryo development in plants ${ }^{8,9}$. Further, TOR is highly expressed in the primary meristems and drives post-embryonic growth in plants ${ }^{8}$. TOR is also essential for the light and sugar-dependent activation of the meristems through E2F transcription factors ${ }^{10-12}$. Light perception leads to the activation of TOR and photomorphogenesis ${ }^{11,13}$. Thus, TOR works as a critical integrator of light and sugar signals to drive post-embryonic growth. TOR is important for the sugar-dependent hypocotyl elongation in dark indicating its role in skotomorphogenesis ${ }^{14}$.

Hyperactivation of TOR results in severe developmental abnormalities in plants and animals $^{9,15}$. Thus, organisms need to maintain optimal TOR activity for normal development. In animals, TORC1 activates the transcription of Sestrins (SESNs) which promotes AMPK leading to the attenuation of TORC1 signaling ${ }^{16,17}$. The GATOR1 complex prevents the hyperactivation of TORC1 during amino acid sufficiency ${ }^{18}$. These negative feedback regulators which prevent TORC1 hyperactivity are absent in the plant lineage ${ }^{19,20}$. Thus, plants possibly have evolved plant-specific mechanisms to moderate TOR signaling. However, such regulators are yet to be discovered in plants. and organogenesis. At the same time, they are exposed to various stress factors in the environment. Rapid sensing of stress signals and induction of stress response pathways are crucial for the survival of plants on land ${ }^{21}$. The high TOR activity can attenuate the stress 
106 response machinery ${ }^{1,22}$; therefore, plants need to maintain a homeostasis between the activities

107 of TOR and stress signaling to balance growth and stress responses.

In this study, we used an interdisciplinary approach to study the importance of mechanisms that regulate TOR signaling to balance growth and stress signaling in plants. Through a data-driven systems biology approach, we identified potential regulatory factors of

112 TOR signaling. We found that one of such factors identified from the screening, FCS-Like Zinc

113 Finger 8 (FLZ8), acts in a negative feedback loop in TOR signaling and maintains basal SnRK1

114 and ABA signaling. This regulation was found to be important in moderating growth and rapid 115 activation of stress signaling in plants.

\section{RESULTS}

\section{Negative feedback regulation of TOR signaling improves fitness of plants in a fluctuating environment}

121 How plants regulate TOR activity to rapidly adjust their growth according to the fluctuations in the

122 environment is not well understood. Negative feedback loops are important in preventing

123 hyperactivation of specific signaling nodes and maintaining homeostasis in cell signaling 124 networks ${ }^{23,24}$. To understand the significance of negative feedback loops in the TOR signaling in 125 plants, we first employed a mathematical modeling approach as it is a powerful tool to decipher 126 the complex and often counter-intuitive dynamics in the cell signaling networks ${ }^{25}$. The mutually 127 antagonistic interaction of TOR and ABA signaling is well understood at the molecular level22; 128 therefore, we developed a simple network model for TOR-ABA signaling to understand how 129 plants regulate TOR signaling under different environmental conditions with and without a 130 negative feedback loop denoted as $X$ (Fig. 1A). We used this model to simulate TOR signaling 131 activation and growth progression under energy sufficiency in a system assumed to have 132 baseline TOR and ABA signaling. The negative feedback loop which is activated by TOR under 133 nutrient sufficiency downregulated TOR activity over time leading to the moderation of growth 134 (Fig. 1B, C). We then asked the relevance of such a feedback loop in regulating stress response 135 in changing environmental conditions. In environment, plants that are growing in favorable 136 conditions with high TOR activity can be suddenly exposed to stress conditions. Therefore, we 137 simulated the ABA signaling activation and stress survivability in response to sudden exposure to 138 stress in a system assumed to have high TOR and baseline ABA signaling. The simulation 139 predicted that the presence of $X$ helps in the rapid activation of the stress signaling module which 140 increased the chance of survival (Fig. 1D, E). Taken together, the simulation of the TOR and 141 stress signaling network under different environmental conditions suggest that the negative 
142 feedback regulation of TOR signaling would facilitate plants in adjusting their growth in a 143 changing environment.

144 Data-driven screening identifies potential negative feedback regulators of TOR signaling

145 To identify the negative feedback regulators of TOR, we adopted a data-driven screening 146 approach. We constructed an Arabidopsis TOR signaling network by integrating all the interactors 147 and phosho-substrates of TOR complex identified from individual and high-throughput omics 148 studies (Fig. 1F and Table S1). We screened for the potential negative feedback regulators from 149 this network which comprises 265 genes. To act as a feedback negative regulator of TOR, the 150 gene needs to be activated by TOR. In Arabidopsis, glucose (Glc) treatment rapidly activates 151 TOR $^{10}$. Therefore, to identify the feedback regulators, we checked the transcriptional regulation of 152 all 265 TOR signaling network genes in Glc treatment using three available transcriptome data 153 sets. Among these genes, the expression of 70 genes was found to be differentially regulated 154 upon short-term Glc treatment in one or more data sets (Fig. 1G). 7 genes were found to be 155 induced in all three data sets. Interestingly, one gene of this category named FLZ8 was found to 156 be a member of the land plant-specific FLZ family implicated in nutrient signaling ${ }^{26}$. FLZ8 157 physically associates with SnRK1 $\alpha$ and $\beta$ subunits and RAPTOR1B subunit of TOR ${ }^{27,28}$. RAPTOR 158 subunit works as a stop-gate of TORC1 activity ${ }^{29}$. Thus, FLZ8 seems to be a potential factor 159 regulating TOR activity in plants through regulating SnRK1-TOR interaction. A close homolog of 160 FLZ8 named FLZ9/MEDIATOR OF ABA-REGULATED DORMANCY 1 (MARD1) also interacts 161 with SnRK1 subunits and RAPTOR ${ }^{28,30}$ (Fig. 1F and Table S1). However, the expression of 162 FLZ9/MARD1 was not induced by Glc treatment (Fig. $1 \mathrm{H}$ ). Further, FLZ9/MARD1 is a primary $163 A B A$ and abiotic stress-responsive gene involved in regulating $A B A$ responses ${ }^{31,32}$. Therefore, we 164 focused on FLZ8 which is a primary Glc-inducible gene for further study. The other 6 genes which 165 were induced in response to Glc treatment in all three transcriptome data sets were found to be 166 heat shock and chaperone proteins and a member of RNA polymerase family (Table S1), thus we 167 did not proceed with them.

169 Sugar sufficiency and TOR induce the expression of $F L Z 8$ by histone modifications

170 To investigate the role of TOR in regulating the expression of $F L Z 8$, we treated seedlings with 171 Torin1, an ATP-competitive inhibitor of TOR ${ }^{33}$. Treatment of Torin1 led to decreased expression 172 of FLZ8 and two TOR-inducible marker genes MINICHROMOSOME MAINTENANCE 3 (MCM3) 173 and E2F TARGET GENE 1 (ETG1) ${ }^{10}$ (Fig. 2A). Further, the expression of FLZ8 and marker 174 genes were found to be increased in two TOR overexpression lines (TOR OE1/GK-166C06 and 
175 TOR OE2/GK-548G07) ${ }^{34}$ (Fig. 2B). These results collectively suggest a positive correlation 176 between TOR signaling and the expression of $F L Z 8$.

To investigate the role of TOR in Glc-dependent induction of $F L Z 8$, we used estradiol 178 (Est)-inducible TOR RNAi line (tor-es1) ${ }^{35}$. The short-term $(3 \mathrm{~h})$ Glc treatment led to a strong 179 induction of $F L Z 8$ in tor-es 1 which was significantly reduced upon the Est treatment (Fig. 2C). It is 180 known that TOR associates with epigenetic factors and regulates histone modifications to control gene expression in response to sugar sufficiency ${ }^{36}$. Histone 3 lysine acetylation (H3KAc) and Histone 3 lysine-4 tri-methylation $(\mathrm{H} 3 \mathrm{~K} 4 \mathrm{me} 3)$ are generally associated with the activation of gene expression ${ }^{37}$. To identify the possible role of TOR and histone modification in regulating the rapid transcriptional upregulation of $F L Z 8$ in response to Glc treatment, we performed a ChIP-qPCR using anti-H3KAc and anti-H3K4me3 antibodies in tor-es1 treated with Glc or Glc and Est. Glc treatment led to enhanced enrichment of $\mathrm{H} 3 \mathrm{KAc}$ and $\mathrm{H} 3 \mathrm{~K} 4 \mathrm{me} 3$ in the upstream regulatory region of $F L Z 8$ with more enrichment in the region closer to the transcriptional start site (TSS) (Fig. 2DF). TOR inhibition through Est treatment reduced the Glc-dependent recruitment of H3KAc (Fig. 2E). Conversely, the recruitment of $\mathrm{H} 3 \mathrm{~K} 4 \mathrm{me} 3$ was not reduced in response to TOR inhibition (Fig. 2F). Taken together, the $\mathrm{qPCR}$ and ChIP-qPCR results indicate that TOR promotes the recruitment of $\mathrm{H} 3 \mathrm{KAC}$ in the promoter region of $F L Z 8$ leading to the activation of its transcription under Glc sufficiency. TOR phosphorylates and activates a CREB-binding protein/p300-type Histone Acetyltransferase in mammals ${ }^{38}$. Its Arabidopsis orthologue Histone Acetyltransferase1 (HAC1) acetylates genes in response to sugar treatment to induce their expression ${ }^{39}$. We found that the induction of $F L Z 8$ level in response to Glc is reduced in hac1 mutants indicating the possible role of HAC1 in regulating the acetylation of FLZ8 promoter in Glc sufficiency (Fig. $2 \mathrm{G}$ ). To test this hypothesis, we performed a ChIP-qPCR using the anti-H3KAc antibody in the hac1 mutants. We found that the Glc-dependent induction of H3KAc in the FLZ8 promoter is significantly reduced in the hac1 mutants (Fig. 2H; Fig. S1). Collectively, our results indicate that the TOR-dependent H3KAc and TOR-independent H3K4me3 in the upstream regulatory regions of $F L Z 8$ induce its expression during sugar and energy sufficiency.

TOR phosphorylates E2Fa, which in turn binds to the promoters of target genes to drive their expression ${ }^{10,40}$. To identify the possible role of E2Fa in regulating the expression of $F L Z 8$, we analyzed the presence of the E2Fa binding sites in the promoter region of FLZ8. We didn't find any typical E2Fa binding sites described previously ${ }^{41}$ in the FLZ8 promoter. However, we found sites with similarity to the typical E2Fa binding sites. Therefore, we performed a ChIPqPCR using the anti-E2Fa antibody to check the binding of E2Fa on the promoter of FLZ8. We used the e2fa mutant as a negative control along with WT and enrichment was analyzed in different promoter regions of FLZ8 and a previously identified E2Fa target $M C M 5^{42}$ (Fig. S2A). In 
210 line with the previous report, specific enrichment of E2Fa was observed in the promoter of MCM5

211 in WT which was significantly reduced in e2fa (Fig. S2B). However, no enrichment was observed

212 in any of the four regions in the $1.6 \mathrm{~kb}$ upstream region of $F L Z 8$ in WT with similar signal

213 intensities in both WT and e2fa (Fig. S2C). These results indicate that E2Fa doesn't bind to the

214 promoter of $F L Z 8$ to drive its expression in Glc sufficiency. Further, we found that the expression

215 of $F L Z 8$ was not significantly altered in the e2fa (Fig. S2d). Taken together, these results clearly

216 indicate that the E2Fa pathway is not involved in the TOR-mediated induction of FLZ8 level

217 during sugar sufficiency.

$218 \quad$ Along with light and sugar, mineral nutrient sufficiency (nitrogen, phosphate, sulfur, etc.)

219 also activates TOR ${ }^{10,13,43,44}$. As FLZ8 levels are rapidly induced in response to short-term Glc

220 treatment in a TOR-dependent manner (Fig. 2), we aimed to identify whether its expression is

221 also induced in response to mineral nutrient (nitrogen, phosphate, iron, sulfur, and potassium)

222 treatment using publically available transcriptome data. We found a clear positive correlation

223 between FLZ8 transcript level and sugar sufficiency (Fig. S3A). However, such correlation was

224 absent between induction of $F L Z 8$ transcript level and the other nutrients. Further, the expression

225 of $F L Z 8$ was found to be upregulated in response to high light treatment and illumination of

226 etiolated plants (Fig. S3B). The expression of FLZ8 is downregulated under sugar starvation

227 which was more pronounced in the overexpression of SnRK1a1, the major kinase of SnRK1 (Fig.

$228 \mathrm{~S} 4 \mathrm{~A})$. Further, SnRK1a1 overexpression abolished the induction of $F L Z 8$ expression in response

229 to sugar treatment (Fig. S4B). Taken together, our results indicate that the Glc-TOR signaling is

230 the specific upstream activator of $F L Z 8$. SnRK1 signaling which operates majorly during sugar

231 starvation suppresses $F L Z 8$ indicating that $F L Z 8$ level is strongly correlated with the changes in

232 the TOR and SnRK1 signaling in plants.

\section{TOR signaling is negatively regulated by FLZ8 in plants}

234 Considering the involvement of $F L Z 8$ in TOR signaling network, we sought to investigate its role 235 in growth and development. We identified two mutants of $F L Z 8$ (Fig. 3A, B). We analyzed the 236 phenotype of these flz8 mutants from the early seedling stage as TOR is an important regulator of 237 seedling development ${ }^{45}$. Cotyledon opening is a crucial TOR-mediated photomorphogenic 238 response during the early seedling development ${ }^{13}$. Light reception promotes auxin signaling 239 which activates the TOR pathway leading to the promotion of translation and cotyledon opening.

240 Defects in TOR and its downstream signaling partners lead to the suppression of cotyledon 241 opening ${ }^{13}$. Under favorable light $\left(60 \mu \mathrm{mol} \mathrm{m}^{-2} \mathrm{sec}^{-1}\right)$, flz8 mutant lines showed enhanced 242 cotyledon opening (Fig. 3C). Further, flz8 seedlings showed enhanced growth with significantly 243 longer primary roots and increased lateral root number, and biomass (Fig. 3D, E). In response to 
244 sugar sufficiency, TOR activates root meristem to drive postembryonic root development. In line

245 with this, TOR inactivation leads to a reduction in the cell cycle activity and root meristem size ${ }^{42}$.

246 We found that $f$ z 8 alleles show a clear increase in the length and diameter of the root meristem

247 (Fig. 3F). Further, in situ monitoring of cell cycle activity using 5-ethynyl-2'-deoxyuridine (EdU; an

248 analog of thymidine) staining identified meristem activity in a larger area in the roots of flz8 lines

249 (Fig. 3G). Therefore, flz8 lines displayed enhanced root growth per day in the growth kinetics

250 analysis (Fig. $3 \mathrm{H}$ ). Similar to the seedling stages, mutants consistently accumulated more

251 biomass in different stages of rosette development (Fig. 3I). Collectively, the phenotypic analysis

252 identified that the loss of $F L Z 8$ causes meristem hyperactivation leading to enhanced growth and 253 biomass.

The phenotypic alterations identified in the flz8 mutants are typically associated with TOR 255 hyperactivation ${ }^{10-12}$. The ribosomal S6 Kinases (S6Ks) are conserved phosho-substrates of TOR 256 and their phosphorylation status is widely used as a readout of TOR activity ${ }^{11,26,35,43}$. 257 Immunoblotting using specific antibodies identified a significant increase in the phosphorylated 258 form of S6Ks in both flz8 lines indicating the enhancement of TOR activity (Fig. 4A). However, the 259 transcript level of TOR complex components and the two paralogs of S6Ks were found to be 260 unaltered indicating that FLZ8 negatively regulates the TOR signaling through regulating its 261 activity (Fig. S5). Changes in the TOR activity can lead to alteration in the sensitivity towards 262 TOR inhibitors ${ }^{46,47}$. We found that $f / z 8$ alleles showed more resistance to TOR inhibitor AZD-8055 263 at moderate concentrations $(0.25$ and $0.5 \mu \mathrm{M})$ (Fig. 4B). TOR promotes sugar-induced hypocotyl 264 elongation in dark ${ }^{14}$. In line with their high TOR activity, flz8 alleles showed enhanced sucrose265 induced elongation of hypocotyls in dark (Fig. 4C). Collectively, these results indicate that loss of 266 FLZ8 leads to TOR hyperactivation leading to enhanced growth in favorable growth conditions.

$T O R$ is highly expressed at the early developmental stages and plays an important role in early seedling development ${ }^{8,10-12}$. We used the $p F L Z 8::$ GUSA lines to monitor the overlap of FLZ8 expression domain with TOR. Interestingly, these lines showed maximum GUS activity at early seedling stages ( 2 and $3 \mathrm{DAG}$ ). The promoter activity progressively decreased in the later

271 stages (Fig. S6A). In adult stages, GUS activity was low in young leaves and it progressively

272 increased in older and senescing leaves which are characteristic of the $F L Z$ family (Fig. S6B) $26,48$.

273 GUS activity was also observed specifically in the developing pollen (Fig. S6C, D). These results

274 suggest that similar to TOR, FLZ8 is also highly expressed during the early seedling stages.

275 FLZ8 associates with critical domains of SnRK1a1 and RAPTOR1B and promotes the 276 SnRK1a1-RAPTOR1B interaction 
277 We sought to investigate the mechanism of FLZ8-mediated negative regulation of TOR. FLZ8 278 physically associates with SnRK1, the prominent negative regulator of TOR signaling. Further, 279 based on the promiscuous interaction of FLZ proteins with SnRK1 and shared interacting 280 proteins, it is hypothesized that FLZ proteins might be working as scaffold proteins that help in the 281 recruitment of phospho-substrates to the SnRK1 complex ${ }^{27,49}$. To understand the possible role of 282 FLZ8 in SnRK1 signaling, we used the yeast-two-hybrid $(\mathrm{Y} 2 \mathrm{H})$ assay to map the interaction site 283 of FLZ8 in SnRK1a1 (Fig. 5A). Mapping of the interaction site identified that FLZ8 interacts 284 strongly with both the catalytic domain (CD) and the regulatory domain (RD). The RD region 285 harbors Ubiquitin-associated domain (UBA) and the $\mathrm{aC}$-terminal domain ( $\mathrm{aCTD}$ ) which is 286 important for the heterotrimeric enzyme complex formation ${ }^{1}$. When the interaction site in the RD 287 region was mapped, FLZ8 specifically interacted with the UBA domain (Fig. 5A). The UBA 288 domain was recently found to be important for the catalytic activity of SnRK1a150. In a large-scale 289 interactome study, FLZ8 was found to be interacting with RAPTOR1B ${ }^{28}$. To confirm this 290 interaction, we used $\mathrm{Y} 2 \mathrm{H}$ assay with an appropriate control experiment which identified the strong 291 interaction of FLZ8 with RAPTOR1B (Fig. 5B). Further, we mapped the interaction site of FLZ8 in 292 RAPTOR1B in which FLZ8 showed specific interaction with Raptor N-terminal CASPase-like 293 (RNC) domain and WD40 repeats located in the C-terminus (Fig. 5C). In mammals, these 294 domains are very critical for TORC1 activity as mutagenesis in these domains abolish the 295 RAPTOR-TOR association ${ }^{51}$. Thus, our mapping analysis identified that FLZ8 makes extensive 296 contacts with SnRK1a1 and RAPTOR1B at multiple sites which are critical for the SnRK1 and 297 TORC1 activity respectively.

The mapping results indicate the potential role of FLZ8 as a scaffold protein which mediates the interaction of SnRK1a1 with RAPTOR1B. To test this hypothesis, we employed a yeast-three-hybrid $(\mathrm{Y} 3 \mathrm{H})$ assay in which along with SnRK1a1 with RAPTOR1B, FLZ8 was expressed under a glucose-repressed and galactose-inducible promoter (GAL1). The expression of FLZ8 in galactose strongly enhanced the interaction of SnRK1a1 with RAPTOR1B which was found to be absent in the cells growing in glucose (Fig 5D, E). Thus, these results identified the molecular function of FLZ8 as a scaffold protein that connects TOR and SnRK1 complexes in 305 plants.

\section{SnRK1 signaling is positively regulated by FLZ8 in plants}

In protein-protein interaction assays, we found that FLZ8 interacts with the UBA domain of SnRK1a1 which is generally involved in preventing protein ubiquitination ${ }^{52}$. Thus, we 309 hypothesized that FLZ8 might be involved in the regulation of SnRK1a1 stability. To test this 310 possibility, we estimated the endogenous level of SnRK1a1 in flz8 mutants at the seedling stage 
311 growing under the normal growth conditions using a specific antibody. Immunoblot analysis 312 identified a clear reduction in the level of endogenous SnRK1a1 in the mutants (Fig. 6A). The 313 transcript level of SnRK1a1 and its paralog $S n R K 1 \alpha 2$ were unaltered in the flz8 mutants (Fig. 314 S7). Thus, FLZ8 promotes SnRK1 signaling through maintaining the level of SnRK1a1. Therefore, we tested whether the decrease in the total pool of SnRK1a1 in the flz8 mutants results in a reduction in the active pool of kinase using an antibody that detects Thr-175 318 phosphorylated form of SnRK1a1. In the immunoblotting, flz8 seedlings growing under normal 319 growth conditions showed a clear reduction in the level of Thr-175 phosphorylated SnRK1a1 (Fig. 320 6B). We further analyzed its effect on endogenous SnRK1 activity using AMARA peptide assay. 321 SnRK1 activity was found to be significantly decreased in the flz8 seedlings in comparison to the 322 WT (Fig. 6C). SnRK1 promotes the expression of Dark Induced 6 (DIN6) by phosphorylating 323 bZIP63 ${ }^{53}$, and Dark Induced $10(D I N 10)^{4}$. The expression of DIN6 and DIN10 is positively 324 correlated with SnRK1 activity and thus widely used as SnRK1 signaling readout ${ }^{4,53-55}$. In line 325 with the reduced SnRK1 activity in the flz8 mutants, we found a significant downregulation of 326 DIN6 and DIN10 transcript levels in the mutants (Fig. 6D). Hyperactivation of SnRK1 signaling 327 leads to ABA hypersensitivity including enhanced inhibition in the primary root growth in response 328 to $A B A$ treatment ${ }^{26,56}$. Therefore, we tested the ABA sensitivity of flz 8 mutants at the seedling 329 stage and found that ABA treatment led to reduced inhibition of primary root growth in the 330 mutants (Fig. 6E). Autophagy is positively mediated by SnRK1 and negatively regulated by TOR 331 signaling ${ }^{57-59}$. Using a specific antibody, we estimated the endogenous autophagy level of $f / z 8$ 332 mutants through assaying the level of ATG8a lipidation with phosphatidylethanolamine (ATG8a$333 \mathrm{PE})$, which is important for the autophagosome formation ${ }^{60}$. The immunoblotting analysis 334 revealed a clear reduction in the level of ATG8a-PE in the flz8.1 line (Fig. S8). Taken together, 335 our results establish FLZ8 as a positive regulator of SnRK1 signaling in normal growth conditions 336 by maintaining the level of SnRK1a1. Loss of FLZ8 leads to reduced endogenous SnRK1a1 level 337 which culminates in reduced SnRK1 activity leading to changes in gene expression, ABA 338 response, and autophagy.

339 The FLZ8-mediated negative feedback module of TOR works as a moderator of growth 340 during sugar sufficiency

341 Our findings show that the level of FLZ8 is a critical factor balancing the TOR-SnRK1 signaling in 342 plants. Induction of FLZ8 during sugar sufficiency by TOR-dependent and independent pathways 343 leads to the maintenance of SnRK1 signaling via stabilization of SnRK1a1 and the promotion of 344 SnRK1a1-RAPTOR1B association. During sugar starvation, SnRK1 signaling downregulates the 
345 FLZ8 level indicating that this module mainly operates in TOR-SnRK1 signaling during sugar

346 sufficiency conditions. To better understand the importance of this negative feedback module on

347 TOR-SnRK1 signaling and growth, we developed a dynamic network model according to our

348 results (Fig. S9A). Using this model, we simulated the effect of FLZ8-mediated regulation on

349 TOR, SnRK1, and biomass accumulation in favorable growth conditions (i.e. energy sufficiency).

350 Simulation of the network under energy sufficiency predicted that FLZ8-mediated regulation

351 moderates TOR signaling and growth by keeping a basal level of SnRK1 signaling (Fig. S9B-D).

352 These results are consistent with our experimental results in which we found hyperactivation of

353 TOR signaling and reduction in the basal SnRK1 signaling in the flz8 mutants growing in optimal

354 light and sugar (30 mM Suc) level (Fig. 4 and 6). To further clarify the role of FLZ8 in sugar

355 availability-dependent growth, we transferred the 5DAG WT, flz8.1, tori (tor 35-7) seedlings to

356 sugar starvation ( $8 \mathrm{mM} \mathrm{Suc}$ ) and sugar abundant (60 mM Suc) growth regimes and the effect

357 was assessed after 5 days. The growth and biomass of all lines were comparable to WT at $8 \mathrm{mM}$

358 Suc indicating the major role of TOR and FLZ8 in regulating growth under sugar sufficiency (Fig.

359 S9E and F). However, significant differences in growth and biomass were observed at $60 \mathrm{mM}$

360 Suc. Consistent with the role of TOR signaling in plant growth and biomass accumulation in

361 nutrient-rich conditions, the tori line showed reduced overall growth and biomass accumulation

362 compared to WT. In comparison, flz8.1 showed more vigorous growth with more biomass in 60

$363 \mathrm{mM}$ Suc (Fig. S9E and F). Taken together, the simulation and experimental results establish

364 FLZ8 as a critical regulator of sugar-dependent growth through modulating the TOR-SnRK1

365 signaling.

366 The FLZ8-mediated negative feedback module of TOR helps in the rapid induction of 367 SnRK1 and ABA signaling during stress

368 Our results indicate that loss of FLZ8 leads to the downregulation of basal SnRK1 signaling which

369 culminates in TOR hyperactivation and negative regulation of autophagy. SnRK1 signaling and

370 autophagy are intimately linked with stress signaling in plants ${ }^{54,61}$. Further, TOR hyperactivation is

371 associated with the downregulation of ABA signaling ${ }^{22}$. Therefore, we hypothesized that the

372 FLZ8-mediated balancing of TOR-SnRK1 signaling would be linked with plants' ability to respond

373 to stress. To test this, we developed a dynamic network model of TOR-SnRK1 signaling in stress

374 conditions (Fig. 7A). We simulated this network to understand how plants growing in favorable

375 conditions respond to sudden exposure to stress with and without the FLZ8-mediated signaling

376 network. The simulation predicted that FLZ8 helps in the rapid activation of SnRK1 and stress

377 signaling leading to enhanced survival (Fig. 7B-D). To test these predictions, we first studied how

378 SnRK1 signaling is regulated to sudden exposure to osmotic and salt stress. We estimated DIN6

379 expression as it is widely used to monitor transient and physiologically relevant SnRK1 
380 signaling ${ }^{4,53-55}$. Osmotic and salt stress treatments led to rapid induction of DIN6 expression at $1 \mathrm{~h}$ 381 which was downregulated at 3h indicating a rapid activation of SnRK1 signaling under stress. 382 This induction of DIN6 expression at $1 \mathrm{~h}$ was significantly attenuated in the flz8 mutant (Fig. 7E 383 and F). Thus, FLZ8 module which helps to maintain a basal level of SnRK1 activity under normal 384 growth conditions helps in the rapid activation of SnRK1 signaling under stress.

$A B A$ and SnRK1 show interdependence in signaling activation and activate a large set of common genes ${ }^{54,62,63}$. Loss of $F L Z 8$ led to changes in TOR-SnRK1 signaling and ABA sensitivity indicating the perturbation in the ABA-mediated stress signaling. To test this, we estimated the induction of ABA-marker genes Responsive to Desiccation 29A (RD29A) and Responsive to Desiccation 29B (RD29B) in short-term osmotic and salt stress treatments in the flz8 mutant. The stress treatments rapidly induced the expression of $R D 29 A$ and $R D 29 B$ at $1 \mathrm{~h}$ which was found to be significantly attenuated in flz8 mutant (Fig. 7G). Further, when exposed to osmotic and salt stress, the mutant showed more pronounced inhibition in root growth and fresh weight in comparison to the WT (Fig. $7 \mathrm{H}$ and I). Taken together, the expression and physiological analysis confirm the simulation results establishing the role of FLZ8 module for the timely activation of stress signaling machinery (Fig. S10).

\section{DISCUSSION}

397 In this study, we identified a feedback regulatory mechanism involved in maintaining optimal level 398 of TOR signaling in plants, which is critical for adjusting growth according to the environmental conditions. Once the TOR signaling is activated in response to sugar sufficiency ${ }^{10,12}$, how plants moderate TOR signaling to maintain signaling homeostasis is not understood. We report that 401 FLZ8, a land plant-specific zinc finger protein 27,30 is a critical regulator of TOR during sugar 402 sufficiency operating in a negative feedback loop and this regulation was found to be crucial in moderating growth and rapid activation of stress signaling in plants (Fig. S10).

The reciprocal interaction of TOR and $A B A$ signaling regulates growth and stress responses in plants ${ }^{22}$. However, this mutually antagonistic interaction operates either when plants are under nutrient sufficiency (i.e. high TOR activity leading to the suppression of ABA signaling) or stress (i.e. activation of $A B A$ signaling leading to the suppression of TOR activity). In opisthokonts, TOR signaling is under tight negative feedback regulation in nutrient sufficiency ${ }^{16,18}$. As these regulatory proteins are absent in the plant lineage, we reasoned that plant might have evolved distinct regulatory mechanisms for adjusting their growth in the fluctuating environment on land. To test this, using the information on TOR-ABA signaling ${ }^{22}$, we modeled the potential 414 fitness in a changing environment. 
415 The high-throughput interaction and phosphoproteomics analyses identified the plant 416 TOR signaling network in high resolution ${ }^{28,64}$. We integrated other experimental data with this to 417 develop an updated network of TOR signaling in plants. Sugar level is one of the primary 418 endogenous signals connected to TOR activity in plants ${ }^{10,12}$. Therefore, we screened the 419 response of 265 TOR signaling network genes in Glc treatment to find potential negative 420 feedback regulatory proteins. 7 genes were found to have strong correlation between 421 transcriptional induction and Glc sufficiency. Among these genes, we focused our work on FLZ8 422 based on its interaction with both RAPTOR1B and SnRK1 subunits ${ }^{27,28}$. A paralog of FLZ8 named 423 FLZ9/MARD1 also interacts with RAPTOR1B and SnRK1 subunits ${ }^{28,30}$. However, its expression 424 was not induced by sugar and it is reported to be a primary ABA-inducible gene ${ }^{31}$.

Our detailed analysis identified the involvement of two distinct histone modifications in the induction of FLZ8 in Glc sufficiency. The enrichment of H3KAc was found to be mediated through 428 the TOR and HAC1 pathway and H3K4me3 enrichment was found to be independent of TOR 429 signaling. Further, FLZ8 expression was found to have a clear positive correlation with Suc and 430 light availability. However, the expression of $F L Z 8$ didn't show any positive correlation with the 431 changes in the level of micronutrients which are also linked with TOR signaling in plants ${ }^{45}$. Thus, 432 the sugar production through photosynthesis is a primary endogenous signal which enhances the 433 FLZ8 level through TOR-dependent and independent pathways. Further, SnRK1 activation under 434 sugar starvation suppresses the level of FLZ8. Collectively, our results establish that the FLZ8 435 level is highly sensitive to changes in the cellular sugar level mediated through TOR-SnRK1 436 signaling and other independent mechanisms.

Mutants of FLZ8 showed TOR hyperactivity in favorable conditions which led to accelerated developmental responses such as the early opening of cotyledons, increased meristem size and activity. This led to enhanced growth and biomass in mutants. Strikingly, this phenotype was found to be strongly correlated with sugar status. The growth acceleration was

443 was completely abolished when grown in sugar-limited (8mM Suc) regime. Taken together, these

444 findings suggest that FLZ8 is a critical regulator of sugar-dependent growth through moderating 445 signaling. The overlapping expression domain of FLZ8 with TOR indicates that the TOR-FLZ8 446 module operates in the early seedling development to maintain the balance of TOR activity in 447 plants.

We found that FLZ8 moderates TOR signaling through promoting SnRK1 signaling by

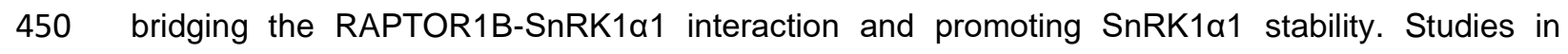


451 different eukaryotes identified that phosphorylation of RAPTOR by AMPK/SnRK1 is a conserved 452 mechanism for the downregulation of TORC1 activity,65. In plants, SnRK1a1 interacts and

453 phosphorylates RAPTOR1B ${ }^{66}$. However, the molecular consequences of this phosphorylation in 454 TOR signaling are yet to be identified. Nonetheless, phosphoproteomics analysis identified 455 constitutive high phosphorylation of Ribosomal Protein S6, a major phosphorylation-target of TOR 456 signaling in snrk1a mutant ${ }^{66}$. Thus, similar to other eukaryotes, the SnRK1a1 and RAPTOR1B 457 interaction results in the phosphorylation of the latter and possibly leads to the downregulation of 458 TOR activity. FLZ8 acts as a scaffold protein which promotes this association causing 459 downregulation of TOR activity.

We found that FLZ8 interacts with the UBA domain of SnRK1a1. A recent report identified that UBA in SnRK1a1 promotes the phosphorylation of SnRK1 by activating kinases and helps in maintaining the kinase activity for longer duration ${ }^{50}$. In general, the UBA domain 464 interacts with ubiquitin and prevents protein degradation ${ }^{52}$. In line with this, loss of $F L Z 8$ led to downregulation of the SnRK1a1 level. The reduction in the SnRK1a1 led to depleted level of SnRK1 active pool and reduced SnRK1 activity in flz8 mutants. Thus, FLZ8 is involved in enhancing the stability of SnRK1a1 possibly by preventing ubiquitination leading to negative regulation of TOR.

The $F L Z$ genes are part of the land-plants specific set of genes ${ }^{27,30}$. On land, plants are more exposed to adverse environmental conditions. The acquisition of novel genes and the 472 evolution of new regulatory mechanisms was critical in the terrestrialization of plants ${ }^{21}$. 473 Simulations identified that FLZ8 prevents hyperactivation of TOR and keeps a basal level of 474 SnRK1 signaling during sugar sufficiency. Supporting this, loss of $F L Z 8$ led to pronounced growth 475 in sugar-rich growth conditions. Further, we hypothesized that through this regulation, FLZ8 might 476 be involved in the regulation of stress responses in plants. Simulations suggested that the FLZ8 477 module helps plants in the rapid activation of SnRK1 and stress signaling when exposed to 478 stress. We tested this hypothesis using osmotic and salt stress conditions as SnRK1 and ABA 479 signaling are involved in regulating stress responses under these conditions ${ }^{4,22,67}$. Analysis of the 480 marker gene expression identified the role of FLZ8 in the rapid (1h) induction of SnRK1 and ABA 481 signaling in stress. As a result, the mutant was hypersensitive to moderate osmotic and salt 482 stress treatments. Thus, FLZ8 enables the cooption of TOR-SnRK1 signaling in plants to deal 483 with the sudden changes in the environment (Fig. S9).

\section{METHODS}




\section{Plant materials and growth conditions}

The Arabidopsis thaliana Columbia-0 (Col-0) ecotype was used for all experiments unless stated otherwise. The SnRK1a1 OE2 line is in the Landsberg erecta (Ler) background ${ }^{4}$. Therefore, Ler was used as the wildtype control in the experiments with SnRK1a1 OE2. The TOR OE1 (GK166C06), TOR OE2 (GK-548G07), tor-es1, tor 35-7, e2fa, hac1-2, hac1-3, hac1-6, and SnRK1a1 OE2 lines were described previously $4,10,34,35,68$. The T-DNA insertion lines of FLZ8 (flz8.1: SALK_129608; flz8.2: SALK_137194C) obtained from ABRC were screened and homozygous lines were identified through PCR-based genotyping method. Insertion in flz8.1 and flz8.2 was found to be in the first exon after $675^{\text {th }}$ and $671^{\text {st }}$ nucleotide, respectively. The primers used are listed in Table S2.

The seeds were surface sterilized and stratified for 2 days in the dark at $4^{\circ} \mathrm{C}$. The seeds were grown on 0.5X Murashige \& Skoog medium (MS; pH: 5.7) with $30 \mathrm{mM}$ Suc (Sigma-Aldrich) and $0.8 \% \mathrm{w} / \mathrm{v}$ agar (Himedia Laboratories, India) in square Petri plates. This composition was used for all experiments unless specified. The experiments on adult stages were performed in pots filled with agro peat and vermiculite (3:1). The plants were cultivated in a climate-controlled growth room under $16: 8 \mathrm{~h}$ photoperiod at $22^{\circ} \mathrm{C}$ and a light intensity of $60 \mu \mathrm{mol} \mathrm{m}^{-2} \mathrm{sec}^{-1}$.

\section{Data-driven screening}

The genes which are involved in the TOR signaling network were retrieved from individual and high-throughput studies directly and through the protein-protein interaction repository BioGRID ${ }^{69}$. The data was manually curated for eliminating repeats and the refined network was visualized in Cytoscape (v3.7.2) $)^{70}$. The transcriptome data was retrieved from Genevestigator ${ }^{71}$ and heatmap visualization and hierarchical clustering (Pearson correlation algorithm with average linkage clustering) was performed in MultiExperiment Viewer (MeV, v4.8) ${ }^{72}$.

\section{Mathematical modeling}

The regulatory networks are modeled using a set of ordinary differential equations (ODE's). MATLAB's ODE solver ode45 was used to solve the Initial Value Problem (IVP) described in the equation. A detailed account of the regulatory network analysis, computational method and MATLAB codes are given as Supplementary methods.

\section{Phenotypic analyses}

The Col-0 and flz8.1 and flz8.2 lines were grown on 0.5X MS medium with $30 \mathrm{mM}$ Suc. The cotyledon angle was measured at 3 DAG. The seedlings were photographed with SteREO Discovery.V12 (Zeiss) stereo microscope and the angle was measured with ImageJ (NIH, USA). The meristem size and diameter were measured at 5 DAG. The seedlings were incubated for 2 min in clearing solution (chloral hydrate: water: glycerol in 8:3:1 ratio). The images were taken using the Axiolmager M2 Imaging System (Zeiss) and meristem size and diameter were measured with ImageJ (NIH, USA). The fresh weight, primary root length and lateral root number were quantified at 10 DAG.

For the root growth kinetics assay, the seedlings were grown on 0.5X MS medium in standard growth conditions and root growth was marked every day from $3^{\text {rd }}$ day onwards for 10 days. The day-wise growth was measured with ImageJ (NIH, USA). For the phenotypic analysis at the adult stages, the Col- 0 and flz8 mutant lines were directly grown on pots and photographs and biomass measurements were taken at 15, 20, 25 and 30 DAG.

\section{AZD8055 sensitivity assay}


529 The 5 DAG Col-0 and flz8 seedlings were transferred to $0.5 \mathrm{X}$ MS medium with 0.25 , 0.5 , and 1 $530 \mu \mathrm{M}$ or without AZD8055. The seedlings were grown for another 5 days at the standard growth 531 conditions and fresh weight was measured.

\section{$532 \quad$ ABA sensitivity assay}

The 5 DAG Col- 0 and flz8 seedlings were transferred to $0.5 \mathrm{X}$ MS medium with 0 and $5 \mu \mathrm{M} \mathrm{ABA}$. The seedlings were grown for another 5 days at the standard growth conditions and root length was measured.

\section{Measurement of sugar-induced hypocotyl elongation in dark}

The 5 DAG Col-0 and flz8 seedlings growing on 0.5X MS medium with $30 \mathrm{mM}$ Suc were kept in darkness in standard growth conditions. The plates were photographed at $0,1,2$, and 3 days of dark treatment and hypocotyl length was measured.

\section{Measurement of sugar-regulated biomass accumulation}

The 5 DAG Col-0, flz8.1, and tor 35-7 seedlings growing on 0.5X MS medium with $30 \mathrm{mM}$ Suc were transferred to $0.5 \mathrm{X}$ MS medium with $60 \mathrm{mM}$ Suc and grown at standard growth conditions for another 5 days. Simultaneously, a control experiment was performed in which seedlings grown on 0.5X MS medium with $30 \mathrm{mM}$ Suc were transferred to $8 \mathrm{mM}$ Suc.

\section{Abiotic stress treatments}

The 5 DAG Col- 0 and flz8 seedlings growing on 0.5X MS medium with $30 \mathrm{mM}$ Suc were transferred to osmotic stress (0.5X MS, $100 \mathrm{mM}$ mannitol, $30 \mathrm{mM} \mathrm{Suc}$ ) and salt stress (0.5X MS, $50 \mathrm{mM} \mathrm{NaCl}, 30 \mathrm{mM}$ Suc) medium. The root growth was measured using Image $(\mathrm{NIH}, \mathrm{USA})$ and fresh weight was measured using a microbalance (Sartorius, Germany).

\section{Measurement of root meristem activity}

Edu (5-ethynyl-2'-deoxyuridine) staining was performed using Click-iT® EdU AlexaFluor ${ }^{\circledR} 647$ Imaging Kit as per the kit protocol (Invitrogen). The 5 DAG Col-0 and flz8 seedlings grown on $0.5 \mathrm{X}$ MS medium with $30 \mathrm{mM}$ Suc were fixed in the fixation reagent (1X PBS with $3.7 \%$ formaldehyde and $0.5 \%$ Triton-X) for $30 \mathrm{~min}$ in a vacuum chamber. After fixing, the seedlings were washed three times in washing buffer (1X PBS with 3\% BSA) and incubated in the reaction cocktail for $30 \mathrm{~min}$ in the dark. After the incubation, seedlings were washed with washing buffer followed by a wash in 1X PBS. The images were taken with Axiolmager M2 Imaging System (Zeiss) (Excitation: $650 \mathrm{~nm}$; Emission: $670 \mathrm{~nm}$ ).

\section{GUS assay}

The $p F L Z 8:: G U S A$ line ${ }^{32}$ was used for the GUS assay. The GUS assay was performed as reported previously ${ }^{73}$ with $3 \mathrm{~h}$ incubation in GUS buffer. The photographs were taken with Axiolmager M2 Imaging System (Zeiss) and SteREO Discovery.V12 (Zeiss) stereo microscopes.

\section{Chemical treatments and sample preparation for qRT-PCR}

For Torin 1 treatment, the Col-0 seedlings were grown on $0.5 \mathrm{X}$ MS solid medium with $30 \mathrm{mM}$ Suc for 10 days. The 10 DAG seedlings were transferred to $0.5 \mathrm{X}$ MS and $30 \mathrm{mM}$ Suc liquid medium with and without Torin $1(10 \mu \mathrm{M})$ in a 6-well plate and incubated at $22^{\circ} \mathrm{C}$ with shaking $(100 \mathrm{rpm})$ for $3 \mathrm{~h}$ and samples were frozen in liquid nitrogen and stored at $-80^{\circ} \mathrm{C}$. The TOR OE lines, e2fa, flz8.1 and flz8.2 were grown along with Col-0 for 10 days on $0.5 \mathrm{X}$ MS solid medium and samples were harvested for qRT-PCR. 
570 For the sugar starvation treatment, the Ler and SnRK1a1 OE2 line were grown for 5 days in $0.5 \mathrm{X}$ 571 MS solid medium with $30 \mathrm{mM}$ Suc. These seedlings were incubated for $24 \mathrm{~h}$ in dark in $0.5 \mathrm{X}$ MS 572 liquid medium without Suc in a 6-well plate at $22^{\circ} \mathrm{C}$ with shaking $(100 \mathrm{rpm})$. One batch of the 573 sample was harvested after $24 \mathrm{~h}$ of starvation and used for qRT-PCR. The other batch was 574 divided into two groups and in one group, the Suc-free medium was replaced by the medium with $57530 \mathrm{mM}$ Suc. Both Suc-free and Suc-replenished samples were harvested after $3 \mathrm{~h}$ of incubation 576 at $22^{\circ} \mathrm{C}$ in dark with shaking at $100 \mathrm{rpm}$.

\section{RNA extraction and qRT-PCR analysis}

578 The RNA isolation was performed by the TRIzol method using RNeasy Plant Mini Kit (Qiagen). 579 The RNA quality and quantity were assessed by NanoDrop 2000 spectrophotometer 580 (ThermoFisher Scientific). Equal quantity of RNA was used for cDNA preparation using the HighCapacity cDNA Reverse Transcription Kit (ThermoFisher Scientific). The qRT-PCR analysis was performed with 1:20 diluted cDNA samples using PowerUp SYBR Green Master Mix (ThermoFisher Scientific) on Step One Plus or ViiA 7 Real-Time PCR System (ThermoFisher Scientific). The primers used for qRT-PCR were designed with Primer Express v3.0 software (ThermoFisher Scientific). The primers used for qRT-PCR are listed in Table S2.

\section{Yeast two- and three- hybrid assays}

The full-length and partial constructs were cloned in pGBKT7g (BD), pGADT7g (AD) and pYESDEST52 vectors using Gateway cloning. The primers used for cloning are listed in Table S2. All experiments were performed in $\mathrm{Y} 2 \mathrm{HGold}$ yeast strain as per the manufacturer's protocol (Clontech). The plates and liquid cultures were grown in incubator at $30^{\circ} \mathrm{C}$ with agitation $(200$ rpm) for liquid cultures.

The transformation was performed using the EZ-Yeast transformation kit (MP Biomedicals). Before the experiments, the auto-activation and toxicity of the prepared BD constructs were tested by spotting an equal amount of yeast culture carrying different constructs on single dropout (SDO) plates supplemented with 5-Bromo-4-chloro-3-indoxyl-a-D-galactopyranoside (X-a-Gal; working concentration: $40 \mu \mathrm{g} / \mathrm{ml}$ ) (Gold Bio) alone or in combination with the yeast toxin Aureobasidin A (AbA; working concentration: $200 \mathrm{ng} / \mathrm{ml}$ ) (Clonetech). Along with AD and BD construct experiments, a negative control experiment with $B D$ vector and $A D$ construct was also performed. The positive colonies were selected on double drop out medium (DDO; -Trp, -Leu) and equal amount of cells were spotted on DDO (-Trp, -Leu) and Quadruple dropout (QDO; -Trp, -Leu, -His, -Ade) medium supplemented with AbA and X- $\alpha$-Gal. The plates were incubated at $30^{\circ} \mathrm{C}$ for 3 days and photographed.

607

608

In $\mathrm{Y} 3 \mathrm{H}$ experiments, the yeast cells containing $\mathrm{AD}$ and $\mathrm{BD}$ combinations were transformed with pYES-DEST52-FLZ8 or pYES-DEST52 and positive colonies were selected on DDO (-Leu, -Ura) and SDO (-Trp). The positive colonies were selected and grown in DDO (-Leu, -Ura) medium with Glc or Gal as the carbon source. The interaction was tested by spotting equal amounts of cells on DDO (-Leu, -Ura), QDO (-Trp, -Leu, -His, -Ade) and QDO medium with AbA and X- $\alpha-$ Gal $(\mathrm{QDO} / \mathrm{X} / \mathrm{A})$. The plates were incubated at $30^{\circ} \mathrm{C}$ for 3 days and photographed.

\section{Protein extraction and western blotting}

10 DAG seedlings were used for western blotting to detect the endogenous level of the phosphorylated (Phospho-p70 S6 kinase pThr389 antibody; dilution: 1:1000; catalog no.: MA515117, Invitrogen) and total (Ribosomal S6 kinase 1/2 antibody; dilution: 1:2000; catalog no.: AS12 1855, Agrisera) level of S6Ks, phosphorylated (Phospho-AMPKa (Thr172) Antibody; dilution: 1:1000; catalog no.: 2531, Cell Signaling Technology) and total (SNF1-related protein kinase catalytic subunit alpha KIN10 antibody; dilution: 1:2000; catalog no.: AS10 919, Agrisera) 616 level of SnRK1a1, and ATG8 (Autophagy-related protein ATG8 antibody; dilution: 1:2000; catalog 
617 no.: AS14 2811, Agrisera). HSP90-2 antibody (Heat shock protein 90-2 antibody; dilution: 1:5000; 618 catalog no.: AS11 1629, Agrisera) was used as the loading control. Total protein was isolated in 619 ice-cold extraction buffer (137 mM NaCl, $2.7 \mathrm{mM} \mathrm{KCl}, 4.3 \mathrm{mM} \mathrm{Na}_{2} \mathrm{HPO}_{4}, 1.47 \mathrm{mM} \mathrm{KH}_{2} \mathrm{PO}_{4}, 10 \%$ $\mathrm{v} / \mathrm{v}$ glycerol, $0.05 \% \mathrm{v} / \mathrm{v}$ Triton X-100) with $1 / 500(\mathrm{v} / \mathrm{v})$ plant-specific protease inhibitor cocktail and phosphatase inhibitor cocktail 3 (Sigma-Aldrich). The protein concentration was quantified by the Bradford method using Protein Assay Dye Reagent (Bio-Rad) in a POLARstar Omega plate reader (BMG Labtech). An equal amount of protein was boiled in Laemmli buffer for 10 min at $95^{\circ} \mathrm{C}$ and separated on $12.5 \%$ acrylamide gel and blotted on a nitrocellulose membrane (GE healthcare). Blocking was done with $0.5 \% \mathrm{w} / \mathrm{v}$ of BSA in Tris-buffered saline with $0.1 \% \mathrm{v} / \mathrm{v}$ Tween 20 (TBST). The blots were developed using Clarity Western ECL Substrate (Bio-Rad) and visualized by ChemiDoc XRS+ imaging system (Bio-Rad). After the first detection, the membranes were stripped using glycine buffer (200 mM glycine, $3.5 \mathrm{mM} \mathrm{SDS,} \mathrm{0.1 \%} \mathrm{v/v} \mathrm{Tween}$ 20, pH: 2.2). After washing three times with TBST, the stripped membranes were blocked and used for immunodetection with other antibodies as per the protocol detailed above. The blots were quantified by ImageJ (NIH, USA).

\section{SnRK1 activity assay}

The total protein was isolated from equal amount of $(\approx 1 \mathrm{~g}) 10$ DAG seedlings of WT and flz8 lines using ice-cold extraction buffer $(50 \mathrm{mM}$ Tris- $\mathrm{HCl}, \mathrm{pH} 7.5,150 \mathrm{mM} \mathrm{NaCl}, 1 \mathrm{mM}$ EDTA, $0.05 \%$ Triton X-100) supplemented with $1 / 500(\mathrm{v} / \mathrm{v})$ plant-specific protease inhibitor cocktail and phosphatase inhibitor cocktail 3 (Sigma-Aldrich). $20 \mu \mathrm{L}$ (bed volume) Pierce Protein A/G Agarose beads (ThermoFisher Scientific) were gently washed three times for 5 min each in phosphate buffered saline (PBS) and incubated with $1.5 \mu \mathrm{g}$ anti-SnRK1a1 antibody (SNF1-related protein kinase catalytic subunit alpha KIN10 antibody; catalog no.: AS10 919, Agrisera) in $600 \mu \mathrm{L}$ PBS on a rotator shaker for $1 \mathrm{~h}$ at room temperature with gentle shaking. The bead-antibody complex was gently washed three times in protein extraction buffer and incubated in $1 \mathrm{mg}$ total protein for $3 \mathrm{~h}$ at $4^{\circ} \mathrm{C}$ on a rotator with gentle shaking. A negative control experiment with protein extraction buffer incubated with antibody-bead complex was also performed along with the test samples. After incubation, beads were gently washed three times using protein extraction buffer and reconstituted to $20 \mu \mathrm{L}$ using the same buffer. From this, $15 \mu \mathrm{L}$ beads were used for SnRK1 activity assay.

The SnRK1 activity assay was performed employing AMARA peptide as the substrate using the Universal Kinase Activity kit which works on the basis of the activity of a coupling phosphatase which removes the inorganic phosphate from the ADP produced due to kinase activity (R\&D Systems). The experiment was conducted as per the manufacturer's protocol. The OD was determined in a POLARstar Omega plate reader (BMG Labtech) and the relative SnRK1 activity in the mutants was determined with reference to WT. A positive control experiment in which ATP was replaced by ADP $(1 \mathrm{mM})$ was also conducted.

\section{Chromatin immunoprecipitation assay}

The ChIP assays were performed according to the previous protocol with minor modifications ${ }^{74}$. The Col-0 and e2fa mutant lines were grown for 7 days on 0.5X MS medium in standard growth conditions. $1 \mathrm{~g}$ tissue of each genotype was crosslinked through vacuum infiltration for 10 min using the $1 \% \mathrm{v} / \mathrm{v}$ formaldehyde solution supplemented with $0.4 \mathrm{M}$ Suc, $10 \mathrm{mM}$ Tris- $\mathrm{HCl} \mathrm{pH} 8,1$ $\mathrm{mM}$ PMSF and $1 \mathrm{mM}$ EDTA. The cross-linked samples were ground in liquid nitrogen and nuclei were isolated. The sonication was performed in ice cold water using Bioruptor Plus sonication device (40 sec on, $20 \mathrm{sec}$ off for 20 cycles) (Diagenode). The DNA-protein complexes were immunoprecipitated using a serum containing anti-E2Fa antibodies and protein digestion and DNA precipitation were performed as per the previous protocol ${ }^{74}$. The enrichment of target promoter regions was analyzed by qRT-PCR using the primers listed in Table S2. 
665 To analyze sugar and TOR-dependent acetylation and methylation, the tor-es 135 line was grown 666 for 7 days in $0.5 \mathrm{X}$ MS medium. On the $7^{\text {th }}$ day, seedlings were transferred to $0.5 \mathrm{X}$ MS medium 667 supplemented with $10 \mu \mathrm{M}$ estradiol or $10 \mu \mathrm{M}$ DMSO. After 4 days, seedlings were used for sugar 668 starvation for $24 \mathrm{~h}$ followed by Glc treatment $(0$ and $30 \mathrm{mM})$ for $3 \mathrm{~h}$ as described previously $\mathrm{y}^{75}$. A 669 small portion of the sample tissue was used for qRT-PCR analysis depicted in Fig. $1 \mathrm{C} .1 \mathrm{~g}$ 670 seedlings of each treatment were used for ChIP assay using the protocol described in the above paragraph. To analyze sugar and TOR-dependent acetylation and methylation, the hac1 lines were grown for 7 days in $0.5 \mathrm{X}$ MS medium and sugar starvation and Glc treatment were performed as described previously ${ }^{75}$. The anti-H3K $(9+14+18+23+27)$ acetylation (Abcam) and anti-H3K4me3 (Millipore) antibodies were used for immunoprecipitation to study acetylation and methylation, respectively. The enrichment of target promoter regions of $F L Z 8$ was analyzed by qRT-PCR using the primers listed in Table S2.

\section{Statistical analyses}

678 Instant Clue ${ }^{76}$, SigmaPlot v13 Microsoft Excel 2010 were used for graph preparation and

679 statistical analyses. All experiments were performed at least three times unless specified.

\section{DATA AVAILABILITY}

682 The mutant/transgenic lines can be requested from the corresponding authors. The authors 683 confirm that any other data related to the findings of this manuscript are available from 684 corresponding authors upon request.

\section{CODE AVAILABILITY}

686 The MATLAB code used for modeling is given in the 'Supplementary methods' section.

\section{REFERENCES}

1. Jamsheer K, M., Jindal, S. \& Laxmi, A. Evolution of TOR-SnRK dynamics in green plants and its integration with phytohormone signaling networks. J. Exp. Bot. 70, 2239-2259 (2019).

2. González, A. \& Hall, M. N. Nutrient sensing and TOR signaling in yeast and mammals.

3. Lin, S.-C. \& Hardie, D. G. AMPK: Sensing Glucose as well as Cellular Energy Status. Cell EMBO J. 36, 397-408 (2017).

4. Baena-González, E., Rolland, F., Thevelein, J. M. \& Sheen, J. A central integrator of transcription networks in plant stress and energy signalling. Nature 448, 938-942 (2007).

5. Gwinn, D. M. et al. AMPK Phosphorylation of Raptor Mediates a Metabolic Checkpoint. Mol. Cell 30, 214-226 (2008).

6. Nukarinen, E. et al. Quantitative phosphoproteomics reveals the role of the AMPK plant ortholog SnRK1 as a metabolic master regulator under energy deprivation. Sci. Rep. 6, 31697 (2016).
nutrient stress. Nat. Metab. (2020). doi:10.1038/s42255-019-0157-1 
706 8. Menand, B. et al. Expression and disruption of the Arabidopsis TOR (target of rapamycin)

9. Ren, M. et al. Target of Rapamycin Regulates Development and Ribosomal RNA

10. Xiong, Y. et al. Glucose-TOR signalling reprograms the transcriptome and activates Expression through Kinase Domain in Arabidopsis. PLANT Physiol. 155, 1367-1382 (2011).

11. Pfeiffer, A. et al. Integration of light and metabolic signals for stem cell activation at the

12. Li, X. et al. Differential TOR activation and cell proliferation in Arabidopsis root and shoot apexes. Proc. Natl. Acad. Sci. U. S. A. 114, 2765-2770 (2017).

13. Chen, G.-H., Liu, M.-J., Xiong, Y., Sheen, J. \& Wu, S.-H. TOR and RPS6 transmit light signals to enhance protein translation in deetiolating Arabidopsis seedlings. Proc. Natl. Acad. Sci. U. S. A. 115, 12823-12828 (2018).

14. Zhang, Z. et al. TOR Signaling Promotes Accumulation of BZR1 to Balance Growth with Carbon Availability in Arabidopsis. Curr. Biol. 26, 1854-1860 (2016).

15. Beauchamp, E. M. \& Platanias, L. C. The evolution of the TOR pathway and its role in cancer. Oncogene 32, 3923-3932 (2013).

16. Lee, J. H. et al. Sestrin as a feedback inhibitor of TOR that prevents age-related pathologies. Science 327, 1223-8 (2010).

17. Budanov, A. V. \& Karin, M. p53 Target Genes Sestrin1 and Sestrin2 Connect Genotoxic Stress and mTOR Signaling. Cell 134, 451-460 (2008).

18. Jin, G. et al. Skp2-Mediated RagA Ubiquitination Elicits a Negative Feedback to Prevent Amino-Acid-Dependent mTORC1 Hyperactivation by Recruiting GATOR1. Mol. Cell 58, 989-1000 (2015).

19. Nedelcu, A. M. Comparative Genomics of Phylogenetically Diverse Unicellular Eukaryotes Provide New Insights into the Genetic Basis for the Evolution of the Programmed Cell Death Machinery. J. Mol. Evol. 68, 256-268 (2009).

20. Panchaud, N., Péli-Gulli, M.-P. \& De Virgilio, C. SEACing the GAP that nEGOCiates TORC1 activation. Cell Cycle 12, 2948-2952 (2013).

21. de Vries, J. \& Archibald, J. M. Plant evolution: landmarks on the path to terrestrial life. New Phytol. 217, 1428-1434 (2018).

22. Wang, P. et al. Reciprocal Regulation of the TOR Kinase and ABA Receptor Balances Plant Growth and Stress Response. Mol. Cell 69, 100-112.e6 (2018).

23. Nguyen, L. K. \& Kholodenko, B. N. Feedback regulation in cell signalling: Lessons for cancer therapeutics. Semin. Cell Dev. Biol. 50, 85-94 (2016).

24. Brandman, O. \& Meyer, T. Feedback loops shape cellular signals in space and time. Science 322, 390-395 (2008).

744 25. Muraro, D., Byrne, H. M., King, J. R. \& Bennett, M. J. Mathematical Modelling Plant 

Signalling Networks. Math. Model. Nat. Phenom. 8, 5-24 (2013).

26. Jamsheer K, M. et al. FCS-like zinc finger 6 and 10 repress SnRK1 signalling in Arabidopsis. Plant J. 94, 232-245 (2018).

27. Jamsheer K, M. et al. The FCS-like zinc finger scaffold of the kinase SnRK1 is formed by the coordinated actions of the FLZ domain and intrinsically disordered regions. J. Biol. Chem. (2018). doi:10.1074/jbc.RA118.002073

28. Arabidopsis Interactome Mapping Consortium. Evidence for Network Evolution in an Arabidopsis Interactome Map. Science (80-. ). 333, 601-607 (2011).

29. Foster, K. G. et al. Regulation of mTOR Complex 1 (mTORC1) by Raptor Ser ${ }^{863}$ and Multisite Phosphorylation. J. Biol. Chem. 285, 80-94 (2010).

30. Jamsheer K, M., Mannully, C. T., Gopan, N. \& Laxmi, A. Comprehensive Evolutionary and Expression Analysis of FCS-Like Zinc finger Gene Family Yields Insights into Their Origin, Expansion and Divergence. PLoS One 10, e0134328 (2015).

31. He, Y. \& Gan, S. A novel zinc-finger protein with a proline-rich domain mediates ABAregulated seed dormancy in Arabidopsis. Plant Mol. Biol. 54, 1-9 (2004).

32. Jamsheer K, M. \& Laxmi, A. Expression of Arabidopsis FCS-Like Zinc finger genes is differentially regulated by sugars, cellular energy level, and abiotic stress. Front. Plant Sci. 6, 746 (2015).

33. Thoreen, C. C. et al. An ATP-competitive mammalian target of rapamycin inhibitor reveals rapamycin-resistant functions of mTORC1. J. Biol. Chem. 284, 8023-32 (2009).

34. Deprost, D. et al. The Arabidopsis TOR kinase links plant growth, yield, stress resistance and mRNA translation. EMBO Rep. 8, 864-870 (2007).

35. Xiong, Y. \& Sheen, J. Rapamycin and glucose-target of rapamycin (TOR) protein signaling in plants. J. Biol. Chem. 287, 2836-42 (2012).

36. Laribee, R. N. Transcriptional and Epigenetic Regulation by the Mechanistic Target of Rapamycin Complex 1 Pathway. J. Mol. Biol. 430, 4874-4890 (2018).

37. Zhao, Y. \& Garcia, B. A. Comprehensive Catalog of Currently Documented Histone Modifications. Cold Spring Harb. Perspect. Biol. 7, a025064 (2015).

38. Wan, W. et al. mTORC1 Phosphorylates Acetyltransferase p300 to Regulate Autophagy and Lipogenesis. Mol. Cell 68, 323-335.e6 (2017).

39. Chen, Q. et al. WRKY18 and WRKY53 coordinate with HISTONE

ACETYLTRANSFERASE1 to regulate rapid responses to sugar. Plant Physiol. pp.00511.2019 (2019). doi:10.1104/pp.19.00511

40. Sharma, M., Banday, Z. Z., Shukla, B. N. \& Laxmi, A. Glucose-Regulated HLP1 Acts as a Key Molecule in Governing Thermomemory. Plant Physiol. 180, 1081-1100 (2019).

41. Vandepoele, K. et al. Genome-wide identification of potential plant E2F target genes. Plant Physiology 139, 316-328 (2005).

42. Xiong, Y. et al. Glucose-TOR signalling reprograms the transcriptome and activates meristems. Nature 496, 181-186 (2013). 
784 43. Dong, Y. et al. Sulfur availability regulates plant growth via glucose-TOR signaling. Nat. Commun. 8, 1174 (2017).

44. Couso, I. et al. Phosphorus Availability Regulates TORC1 Signaling via LST8 in

45. Shi, L., Wu, Y. \& Sheen, J. TOR signaling in plants: conservation and innovation. Development 145, dev160887 (2018).

46. Chan, T. F., Carvalho, J., Riles, L. \& Zheng, X. F. A chemical genomics approach toward understanding the global functions of the target of rapamycin protein (TOR). Proc. Natl. Acad. Sci. U. S. A. 97, 13227-32 (2000).

47. Montané, M.-H. \& Menand, B. TOR inhibitors: from mammalian outcomes to pharmacogenetics in plants and algae. J. Exp. Bot. 70, 2297-2312 (2019).

48. He, Y. et al. Networking Senescence-Regulating Pathways by Using Arabidopsis Enhancer Trap Lines. PLANT Physiol. 126, 707-716 (2001).

49. Nietzsche, M., Landgraf, R., Tohge, T. \& B??rnke, F. A protein-protein interaction network linking the energy-sensor kinase SnRK1 to multiple signaling pathways in Arabidopsis thaliana. Curr. Plant Biol. 5, 36-44 (2016).

50. Emanuelle, S., Doblin, M. S., Gooley, P. R. \& Gentry, M. S. The UBA domain of SnRK1 promotes activation and maintains catalytic activity. Biochem. Biophys. Res. Commun. 497, 127-132 (2018).

51. Kim, D.-H. et al. mTOR Interacts with Raptor to Form a Nutrient-Sensitive Complex that Signals to the Cell Growth Machinery. Cell 110, 163-175 (2002).

52. Heinen, C., Ács, K., Hoogstraten, D. \& Dantuma, N. P. C-terminal UBA domains protect ubiquitin receptors by preventing initiation of protein degradation. Nat. Commun. 2, (2011).

53. Mair, A. et al. SnRK1-triggered switch of bZIP63 dimerization mediates the low-energy response in plants. Elife 4, (2015).

54. Rodrigues, A. et al. ABI1 and PP2CA phosphatases are negative regulators of Snf1related protein kinase1 signaling in Arabidopsis. Plant Cell 25, 3871-84 (2013).

55. Ramon, M. et al. Default activation and nuclear translocation of the plant cellular energy sensor SnRK1 regulate metabolic stress responses and development. Plant Cell tpc.00500.2018 (2019). doi:10.1105/tpc.18.00500

56. Jossier, M. et al. SnRK1 (SNF1-related kinase 1) has a central role in sugar and ABA signalling in Arabidopsis thaliana. Plant J. 59, 316-328 (2009).

57. Liu, Y. \& Bassham, D. C. TOR Is a Negative Regulator of Autophagy in Arabidopsis thaliana. PLoS One 5, e11883 (2010).

58. Soto-Burgos, J. \& Bassham, D. C. SnRK1 activates autophagy via the TOR signaling pathway in Arabidopsis thaliana. PLoS One 12, e0182591 (2017).

59. Chen, L. et al. The AMP-Activated Protein Kinase KIN10 Is Involved in the Regulation of Autophagy inArabidopsis. Front. Plant Sci. 8, 1201 (2017).

60. Klionsky, D. J. et al. Guidelines for the use and interpretation of assays for monitoring 

autophagy (3rd edition). Autophagy 12, 1-222 (2016).

61. Margalha, L., Confraria, A. \& Baena-González, E. SnRK1 and TOR: modulating growthdefense trade-offs in plant stress responses. J. Exp. Bot. 70, 2261-2274 (2019).

62. Gazzarrini, S., Tsuchiya, Y., Lumba, S., Okamoto, M. \& McCourt, P. The Transcription Factor FUSCA3 Controls Developmental Timing in Arabidopsis through the Hormones Gibberellin and Abscisic Acid. Dev. Cell 7, 373-385 (2004).

63. Tsai, A. Y.-L. \& Gazzarrini, S. AKIN10 and FUSCA3 interact to control lateral organ development and phase transitions in Arabidopsis. Plant J. 69, 809-821 (2012).

64. Van Leene, J. et al. Capturing the phosphorylation and protein interaction landscape of the plant TOR kinase. Nat. Plants 5, 316-327 (2019).

65. Hughes Hallett, J. E., Luo, X. \& Capaldi, A. P. Snf1/AMPK promotes the formation of Kog1/raptor-bodies to increase the activation threshold of TORC1 in budding yeast. Elife 4, (2015).

66. Nukarinen, E. et al. Quantitative phosphoproteomics reveals the role of the AMPK plant ortholog SnRK1 as a metabolic master regulator under energy deprivation. Sci. Rep. 6, 31697 (2016).

67. Jamsheer K, M. et al. The FCS-LIKE ZINC FINGER 6 and 10 are involved in regulating osmotic stress responses in Arabidopsis. Plant Signal. Behav. 1-4 (2019). doi:10.1080/15592324.2019.1592535

68. Heisel, T. J., Li, C. Y., Grey, K. M. \& Gibson, S. I. Mutations in HISTONE ACETYLTRANSFERASE1 affect sugar response and gene expression in Arabidopsis. Front. Plant Sci. 4, 245 (2013).

69. Oughtred, R. et al. The BioGRID interaction database: 2019 update. Nucleic Acids Res. 47, D529-D541 (2019).

70. Su, G., Morris, J. H., Demchak, B. \& Bader, G. D. Biological Network Exploration with Cytoscape 3. Curr. Protoc. Bioinforma. 2014, 8.13.1-8.13.24 (2014).

71. Hruz, T. et al. Genevestigator V3: A Reference Expression Database for the MetaAnalysis of Transcriptomes. Adv. Bioinformatics 2008, 1-5 (2008).

72. Saeed, A. I. et al. [9] TM4 Microarray Software Suite. in Methods in enzymology 411, 134193 (2006).

73. Jefferson, R. A., Kavanagh, T. A. \& Bevan, M. W. GUS fusions: beta-glucuronidase as a sensitive and versatile gene fusion marker in higher plants. EMBO J. 6, 3901-7 (1987).

74. Saleh, A., Alvarez-Venegas, R. \& Avramova, Z. An efficient chromatin immunoprecipitation (ChIP) protocol for studying histone modifications in Arabidopsis plants. Nat. Protoc. 3, 1018-1025 (2008).

75. Mishra, B. S., Singh, M., Aggrawal, P. \& Laxmi, A. Glucose and Auxin Signaling Interaction in Controlling Arabidopsis thaliana Seedlings Root Growth and Development. PLoS One 4, e4502 (2009).

76. Nolte, H., MacVicar, T. D., Tellkamp, F. \& Krüger, M. Instant Clue: A Software Suite for Interactive Data Visualization and Analysis. Sci. Rep. 8, 12648 (2018). 
864

865

866

867

868

869

870

871

872

873

874

875

876

877

878

879

880

\section{1}

882

883

884

885

886

887

888

889

890

891

892

893

894

895

896

897

898

\section{ACKNOWLEDGMENTS}

This work was supported by the Department of Biotechnology (Project Grant BT/PR8001/BRB/10/1211/2013 and NIPGR Core Grant to AL; DBT-Senior Research Fellowship to MS, NIPGR-Junior Research Fellowship to CTM), Department of Science and Technology (INSPIRE Faculty Programme Grant IFA18-LSPA110 to MJK) and University Grant Commission (UGC-Senior Research Fellowship to MS), Government of India. The authors acknowledge NIPGR Confocal Facility for their assistance and DBT-eLibrary Consortium (DeLCON) for providing access to e-resources. We thank Filip Rolland for providing SnRK1a1 OE2 seeds and Dr. Christian Meyer for tor 35-7 seeds. We thank Dr. Manoj Kumar and Dr. Pallavi Agarwal for insightful discussion and constructive criticism of the manuscript.

\section{AUTHOR CONTRIBUTIONS}

MJK and AL conceived the study. MJK, SJ, and AL designed the experiments. MS performed ChIP-qPCR experiments. MS, MJK, and SJ performed physiological experiments. MJK and CTM performed the mutant screening. SS and MJK performed the computational analysis. MJK and SJ performed all other experiments. MJK wrote the paper. SJ, and AL reviewed the paper.

\section{COMPETING INTERESTS}

The authors declare no competing interests

\section{MATERIALS \& CORRESPONDENCE}

The request for materials and any other correspondence should be addressed to Muhammed Jamsheer K or Ashverya Laxmi 
900 Fig. 1: Simulation and data-driven screening for the potential negative feedback regulators of plant TOR signaling.

902 (A) The Network model developed for simulating TOR-ABA signaling with a negative feedback module of TOR (named as $\mathrm{X}$ ). The green arrows indicate a positive influence and red arrows indicate a negative influence. The arrows connecting $X$ to TOR are represented by dotted arrows. (B) and (C) Simulation of TOR signaling activation and growth under a favorable growth condition with and without $X$ module. (D) and (E) Simulation of $A B A$ signaling activation and survivability in response to sudden exposure to stress with and without $X$ module. (F) The Arabidopsis TOR signaling network which includes identified interacting proteins of TORC components (TOR, RAPTOR1, and LST8-1) and phosphosubstrates of TOR. (G) Heat map of TOR signaling network genes differentially regulated in Glc-transcriptome data sets (DS 1-3). (H) Comparison of transcriptional regulation of $F L Z 8$ and $F L Z 9 / M A R D 1$ in response to Glc treatment in the Glc-transcriptome data sets.

Fig. 2: TOR-dependent and -independent pathways direct specific histone modifications to promote the expression of $F L Z 8$ during sugar sufficiency.

(A) Expression of MCM3, ETG1, and FLZ8 in response to Torin $1(10 \mu \mathrm{M})$ treatment (one-way ANOVA, * $p \leq 0.05$, Bonferroni post-hoc test). (B) Expression of MCM3, ETG1, and FLZ8 in TOR overexpression (TOR OE) lines (one-way ANOVA, ${ }^{*} p \leq 0.05$, Bonferroni post-hoc test). (C) Expression of FLZ8 in tor-es1 line (growing in 0 or $10 \mu \mathrm{M}$ estradiol) treated with 0 or $170 \mathrm{mM} \mathrm{Glc} \mathrm{(two-way}$ ANOVA, ${ }^{*} p \leq 0.05$, Bonferroni post-hoc test). (D) Promoter and upstream regulatory regions of $F L Z 8$. The regions (R1 to $\mathrm{R} 4$ ) tested in ChIP-qPCR are indicated. (E) Histone acetylation (H3KAc) status in the upstream region of $F L Z 8$ in tor-es1 line (growing in 0 or $10 \mu \mathrm{M}$ estradiol) treated with 0 or $170 \mathrm{mM}$ Glc (two-way ANOVA, ${ }^{*} p \leq 0.05$, Bonferroni post-hoc test). (F) Histone methylation (H3K4me3) status in the upstream region of $F L Z 8$ in tor-es1 line (growing in 0 or $10 \mu \mathrm{M}$ estradiol) treated with 0 or $170 \mathrm{mM}$ Glc (two-way ANOVA, ${ }^{*} \mathrm{p} \leq 0.05$, Bonferroni post-hoc test). (G) Expression of FLZ8 in hac1 mutant lines treated with 0 or $170 \mathrm{mM}$ Glc $\left(\mathrm{n}=3\right.$ ) (two-way ANOVA, ${ }^{*} \mathrm{p} \leq 0.05$, Bonferroni post-hoc test). $(\mathrm{H})$ Histone acetylation (H3KAc) status in the upstream region of $F L Z 8$ promoter in hac1-3 treated with 0 or 170 mM Glc (two-way ANOVA, * $p \leq 0.05$, Bonferroni post-hoc test). The error bars of bar graphs indicate SE.

\section{Fig. 3: Meristem activity and growth are enhanced in flz8 mutants.}

(A) Gene structure of FLZ8 showing the T-DNA insertion site in flz8.1 and flz8.2 alleles. The position of primer sets (RT1 and RT2) used for qPCR is also shown in the gene structure. (B) Expression of $F L Z 8$ in mutant lines (one-way ANOVA, ${ }^{*} p \leq 0.05$, Bonferroni post-hoc test). (C) Cotyledon angle of WT and flz8 lines at 3 DAG. (D) and (E) Phenotype and measurement of biomass, primary root length, 
and lateral root number of WT and flz8 lines at 10 DAG. (F) Root meristem size (length and diameter) of WT and flz8 lines at 5 DAG. Blue arrow points to the quiescent centre and black arrow points to the junction between meristem and elongation zone (G) Edu (5-ethynyl-2'-deoxyuridine) staining showing root meristem activity of WT and flz8 lines at 5 DAG. $(\mathrm{H})$ Root growth kinetics in WT and $f / z 8$ lines. (I) Measurement of rosette biomass at different rosette stages and phenotype of WT and flz8 lines at 30 DAG (one-way ANOVA, ${ }^{*} p \leq 0.05$, Bonferroni post-hoc test).

\section{Fig. 4: TOR signaling is hyperactivated in flz8 mutants.}

(A) Level of total and phosphorylated S6Ks (Thr-449 in S6K1 and Thr-455 in S6K2) in WT and flz8 mutants at 10 DAG. Relative level of S6K-P was quantified in comparison to total S6K (one-way ANOVA, ${ }^{*} p \leq 0.05$, Bonferroni post-hoc test). (B) Phenotype and fresh weight of WT and mutant lines in different concentrations of TOR inhibitor AZD8055 (two-way ANOVA, ${ }^{*} p \leq 0.05$, Bonferroni post-hoc test). (C) Sucrose-dependent hypocotyl elongation in WT and flz8.1 in response to dark treatment for 1, 2 and 3 days (two-way ANOVA, * $\mathrm{p}$ $\leq 0.05$, Bonferroni post-hoc test).

\section{Fig. 5: FLZ8 promotes the interaction of SnRK1a1 with RAPTOR1B}

(A) Domain organization of SnRK1a1 and mapping of FLZ8-interaction sites in SnRK1a1 by $\mathrm{Y} 2 \mathrm{H}$ analysis. (B) Interaction of FLZ8 with RAPTOR1B. (C) Domain organization of RAPTOR1B and mapping of FLZ8-interaction sites in RAPTOR1B. (D) and (E) Analysis of the interaction of SnRK1 11 and RAPTOR1B in the presence of $\mathrm{FLZ8}$ by $\mathrm{Y} 3 \mathrm{H}$ analysis in glucose and galactose. For $\mathrm{Y} 2 \mathrm{H}$, the cotransformed yeast cells carrying different $A D$ and $B D$ construct combinations were spotted on synthetic double dropout (DDO; -Trp/-Leu) and quadruple dropout medium supplemented with $40 \mu \mathrm{g} / \mathrm{mL}$ X- $\alpha-G a l$ and $200 \mathrm{ng} / \mathrm{mL} A b A$ (QDO/X/A; -Ade/-His/-Leu/-Trp/+ X- $\alpha-G a l /+A b A)$. For $\mathrm{Y} 3 \mathrm{H}$, the cotransformed yeast cells carrying different $A D$ and $B D$ construct combinations along with pYES (GAL1) FLZ8) or pYES (GAL1) growing in glucose or galactose were spotted on DDO medium and weak (QDO) and strong (QDO/X/A) interaction screening medium.

\section{Fig. 6: SnRK1 signaling is attenuated in flz8 mutants.}

(A) Level of SnRK1a1 in WT and flz8 mutants at 10 DAG. Relative level of SnRK1a1 was quantified in comparison to HSP90-2 level (one-way ANOVA, * $p \leq$ 0.05, Bonferroni post-hoc test). (B) Level of phosphorylated SnRK1a1 (Thr-175) in WT and flz8 mutants at 10 DAG. Relative level of SnRK1a1-P was quantified in comparison to HSP90-2 level (one-way ANOVA, ${ }^{*} p \leq 0.05$, Bonferroni posthoc test). (C) SnRK1 activity in 10 DAG flz8 mutants in comparison to WT in AMARA peptide assay (one-way ANOVA, ${ }^{*} p \leq 0.05$, Bonferroni post-hoc test). (D) Expression of SnRK1-activity reporter genes (DIN6 and DIN10) in 10 DAG flz8 mutants in comparison to WT (one-way ANOVA, ${ }^{*} p \leq 0.05$, Bonferroni post- 
981 hoc test). (E) Phenotype and primary root length of WT and flz8 mutants in

982 response to ABA treatment for 5 days (two-way ANOVA, ${ }^{*} p \leq 0.05$, Bonferroni

983 post-hoc test).

984 Fig. 7: The FLZ8-mediated negative feedback promotes stress tolerance 985 through the rapid activation of SnRK1 and stress signaling

986 (A) Network model developed for simulating the TOR-FLZ8-SnRK1 signaling 987 controlling stress response. The green arrows indicate a positive influence and 988 red arrows indicate a negative influence. The signaling networks which connect 989 FLZ8 with other modules are represented by dotted arrows as these networks 990 were absent while modeling the condition 'without FLZ8'. (B)-(D) Simulation of 991 SnRK1 activity, stress response activation and survival under sudden exposure 992 to stress with and without FLZ8. (E) and (F) Induction of DIN6 expression in 993 response to short-term (1 and $3 \mathrm{~h}$ ) osmotic (100 mM mannitol) and salt (50 mM $994 \mathrm{NaCl}$ ) stresses in WT and flz8.1 (two-way ANOVA, * $p \leq 0.05$, Bonferroni post-hoc 995 test). (G) Induction of $R D 29 A$ and $R D 29 B$ (abiotic stress marker genes) 996 expression in response to short-term (1 and $3 \mathrm{~h}$ ) osmotic (100 mM mannitol) and 997 salt $(50 \mathrm{mM} \mathrm{NaCl})$ stress treatments in WT and flz8.1 mutant (two-way ANOVA, $998{ }^{*} \mathrm{p} \leq 0.05$, Bonferroni post-hoc test). (H) Effect of short-term (1 day) and long999 term (6 days) osmotic stress (100 mM mannitol) treatments on root length and 1000 growth in WT and flz8.1 (two-way ANOVA, * $p \leq 0.05$, Bonferroni post-hoc test). 1001 (I) Effect of short-term (1 day) and long-term (6 days) salt stress (50 mM NaCl) 1002 treatments on root length and growth in WT and flz8.1 (two-way ANOVA, * $p \leq$ 1003 0.05, Bonferroni post-hoc test). 
A

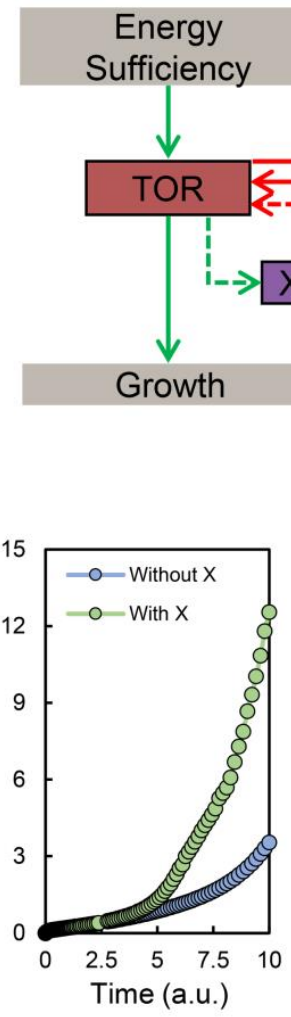

B

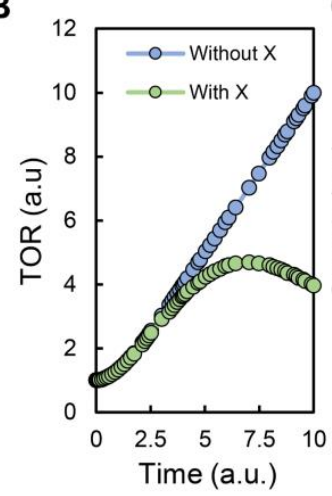

$\mathbf{F}$

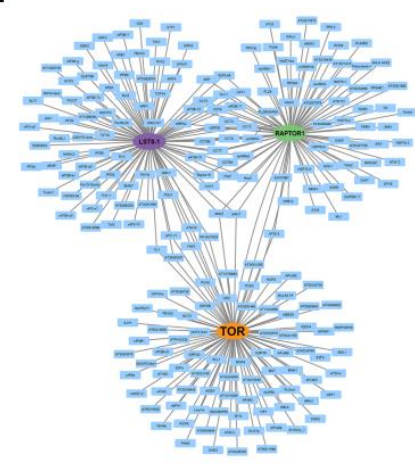

C

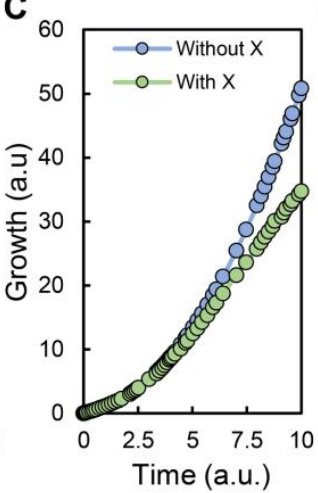

D

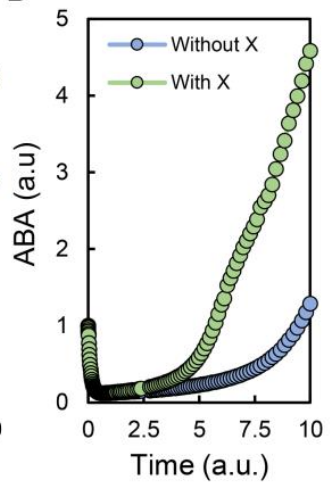

G

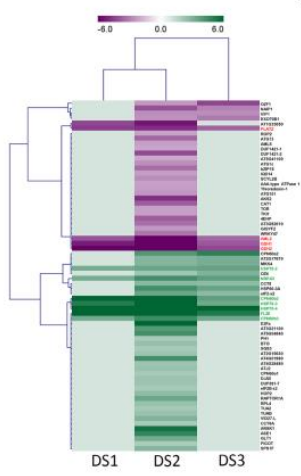

H

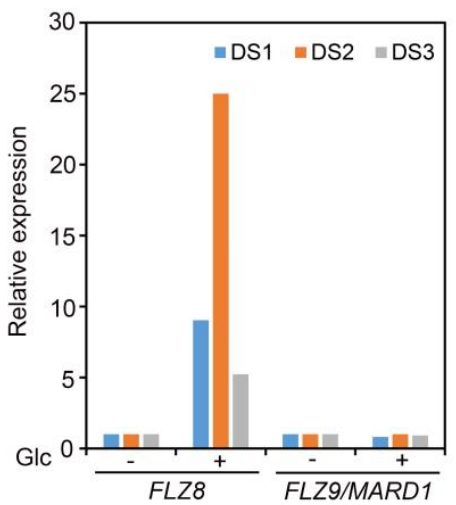

Fig. 1: Simulation and data-driven screening for the potential negative feedback regulators of plant TOR signaling.

(A) The Network model developed for simulating TOR-ABA signaling with a negative feedback module of TOR (named as $\mathrm{X}$ ). The green arrows indicate a positive influence and red arrows indicate a negative influence. The arrows connecting $X$ to TOR are represented by dotted arrows. (B) and (C) Simulation of TOR signaling activation and growth under a favorable growth condition with and without $X$ module. (D) and (E) Simulation of ABA signaling activation and survivability in response to sudden exposure to stress with and without $X$ module. (F) The Arabidopsis TOR signaling network which includes identified interacting proteins of TORC components (TOR, RAPTOR1, and LST8-1) and phosphosubstrates of TOR. (G) Heat map of TOR signaling network genes differentially regulated in Glc-transcriptome data sets (DS 1-3). (H) Comparison of transcriptional regulation of $F L Z 8$ and FLZ9/MARD1 in response to Glc treatment in the Glc-transcriptome data sets. 
A

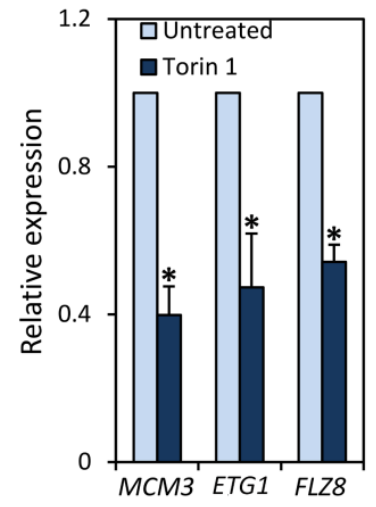

B

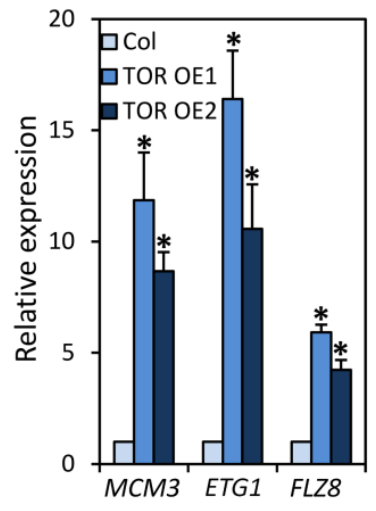

C

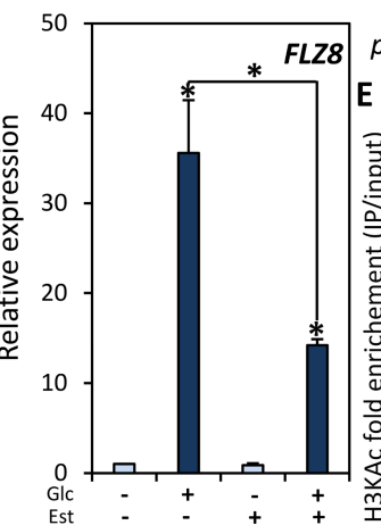

D

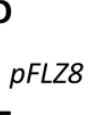

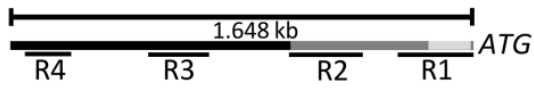

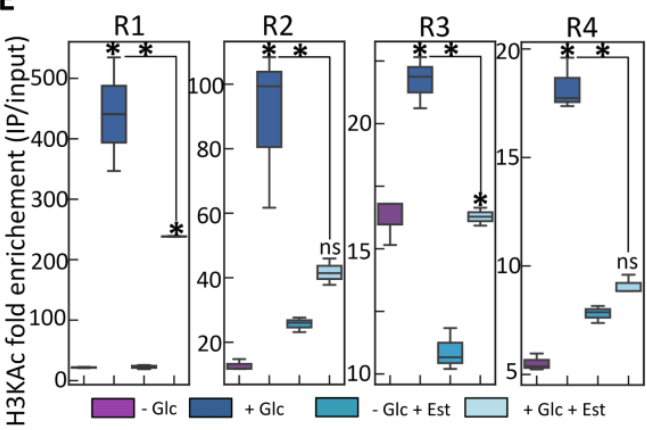

F
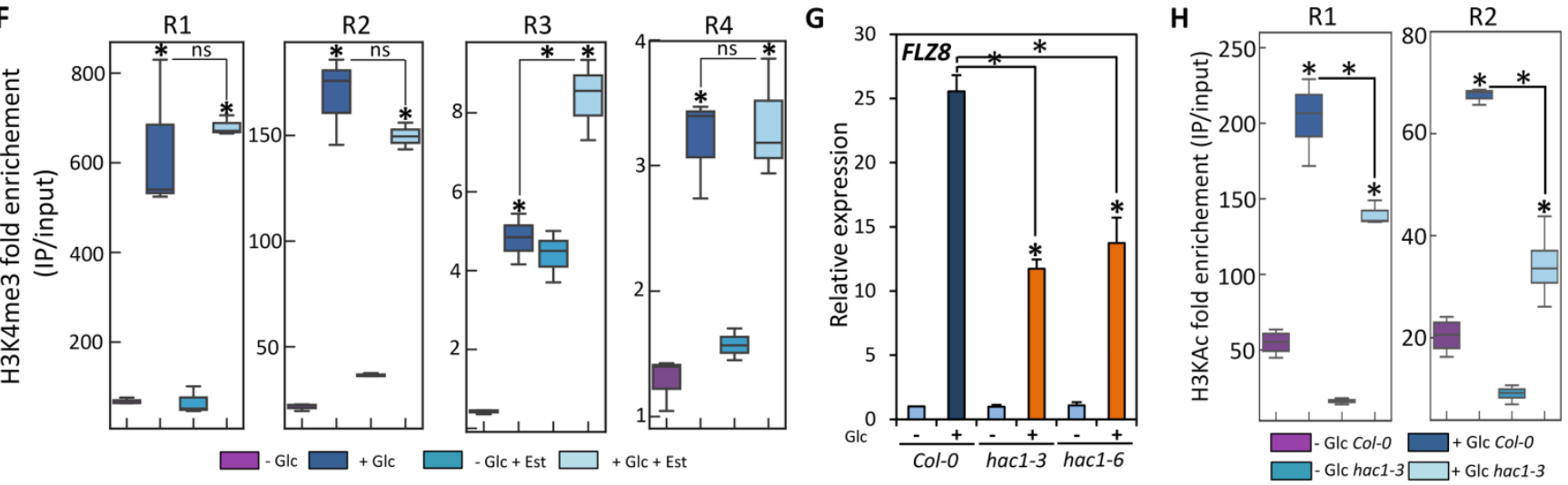

Fig. 2: TOR-dependent and -independent pathways direct specific histone modifications to promote the expression of $F L Z 8$ during sugar sufficiency.

(A) Expression of MCM3, ETG1, and FLZ8 in response to Torin $1(10 \mu \mathrm{M})$ treatment (one-way ANOVA, ${ }^{*} \mathrm{p} \leq$ 0.05, Bonferroni post-hoc test). (B) Expression of MCM3, ETG1, and FLZ8 in TOR overexpression (TOR OE) lines (one-way ANOVA, ${ }^{*} p \leq 0.05$, Bonferroni post-hoc test). (C) Expression of $F L Z 8$ in tor-es 1 line (growing in 0 or $10 \mu \mathrm{M}$ estradiol) treated with 0 or $170 \mathrm{mM} \mathrm{Glc}$ (two-way ANOVA, ${ }^{*} \mathrm{p} \leq 0.05$, Bonferroni post-hoc test). (D) Promoter and upstream regulatory regions of $F L Z 8$. The regions (R1 to R4) tested in ChIP-qPCR are indicated. (E) Histone acetylation $(\mathrm{H} 3 \mathrm{KAC})$ status in the upstream region of $F L Z 8$ in tor-es1 line (growing in 0 or $10 \mu \mathrm{M}$ estradiol) treated with 0 or $170 \mathrm{mM}$ Glc (two-way ANOVA, ${ }^{*} p \leq 0.05$, Bonferroni post-hoc test). (F) Histone methylation (H3K4me3) status in the upstream region of $F L Z 8$ in tor-es1 line (growing in 0 or $10 \mu \mathrm{M}$ estradiol) treated with 0 or $170 \mathrm{mM}$ Glc (two-way ANOVA, ${ }^{*} p \leq 0.05$, Bonferroni post-hoc test). (G) Expression of $F L Z 8$ in hac1 mutant lines treated with 0 or $170 \mathrm{mM} \mathrm{Glc}(\mathrm{n}=3)$ (two-way ANOVA, ${ }^{*} \mathrm{p} \leq 0.05$, Bonferroni post-hoc test). $(\mathrm{H})$ Histone acetylation (H3KAc) status in the upstream region of $F L Z 8$ promoter in hac1-3 treated with 0 or $170 \mathrm{mM}$ Glc (two-way ANOVA, ${ }^{\star} p \leq 0.05$, Bonferroni post-hoc test). The error bars of bar graphs indicate SE. 

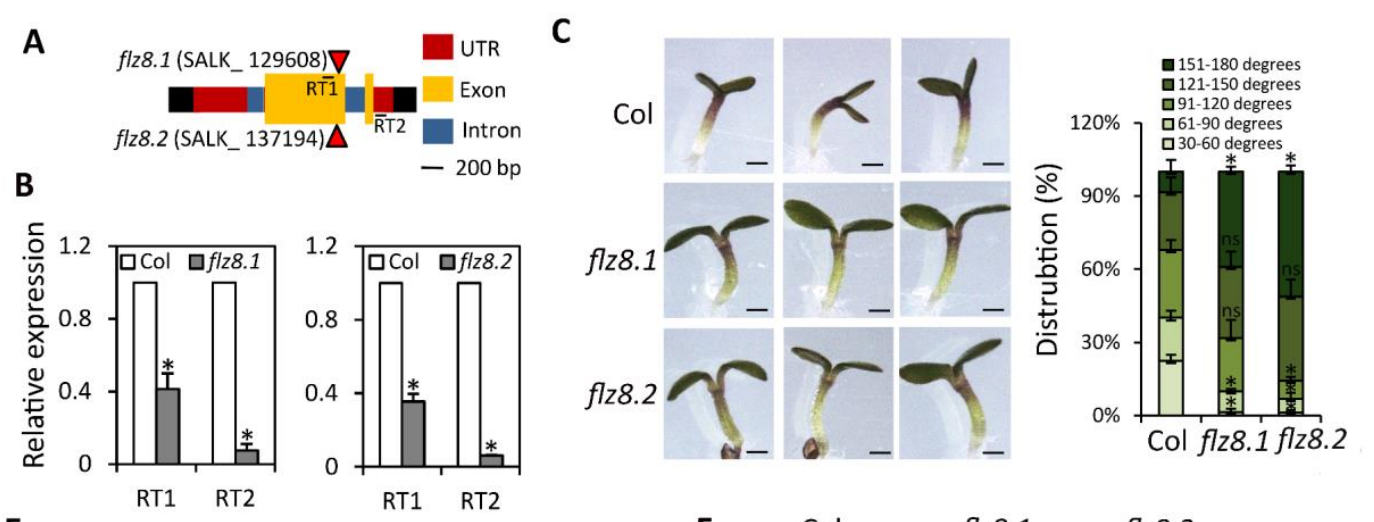

D

E

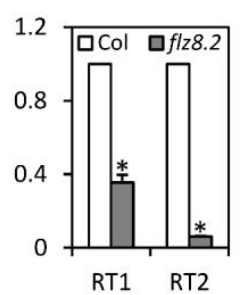

F
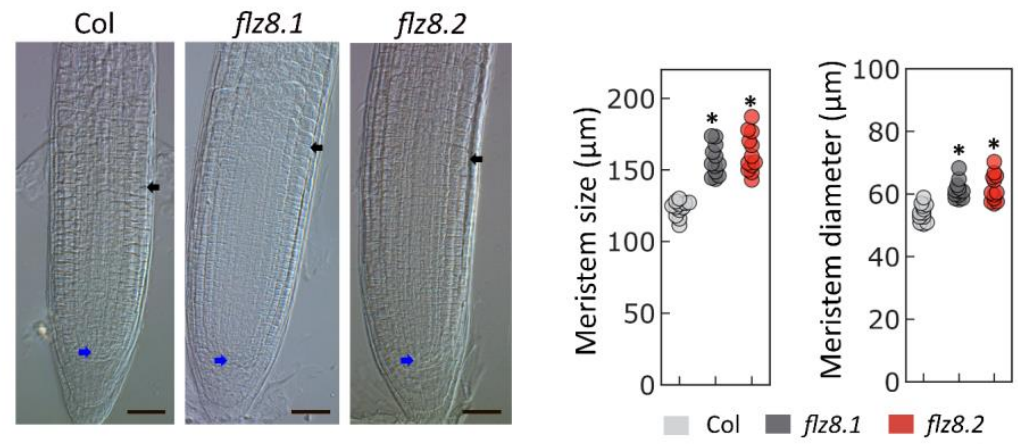

G
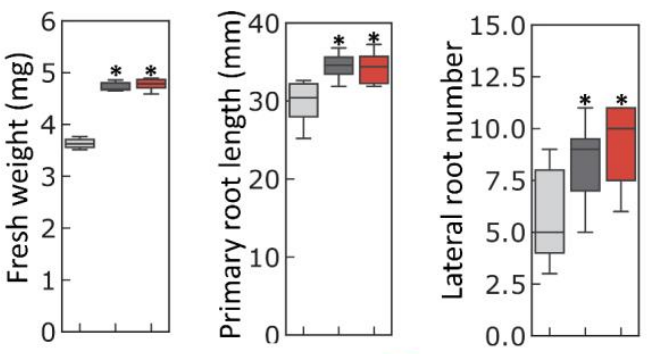

Col $\square$ flz8.1 flz8.2

H

Col

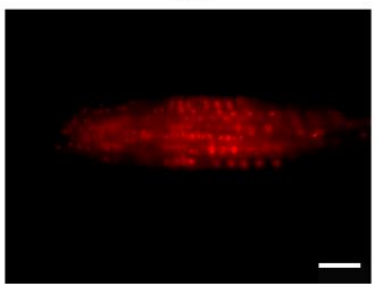

flz8.1

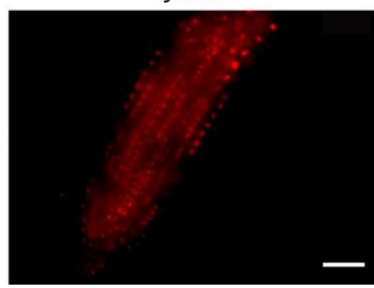

flz8.2

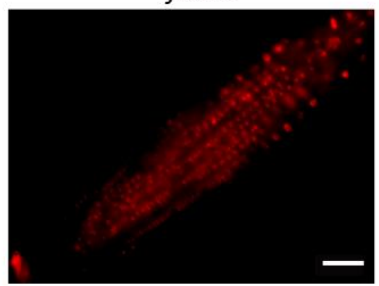

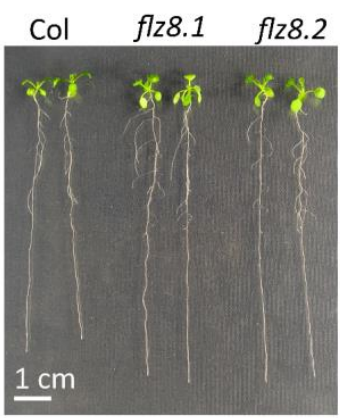
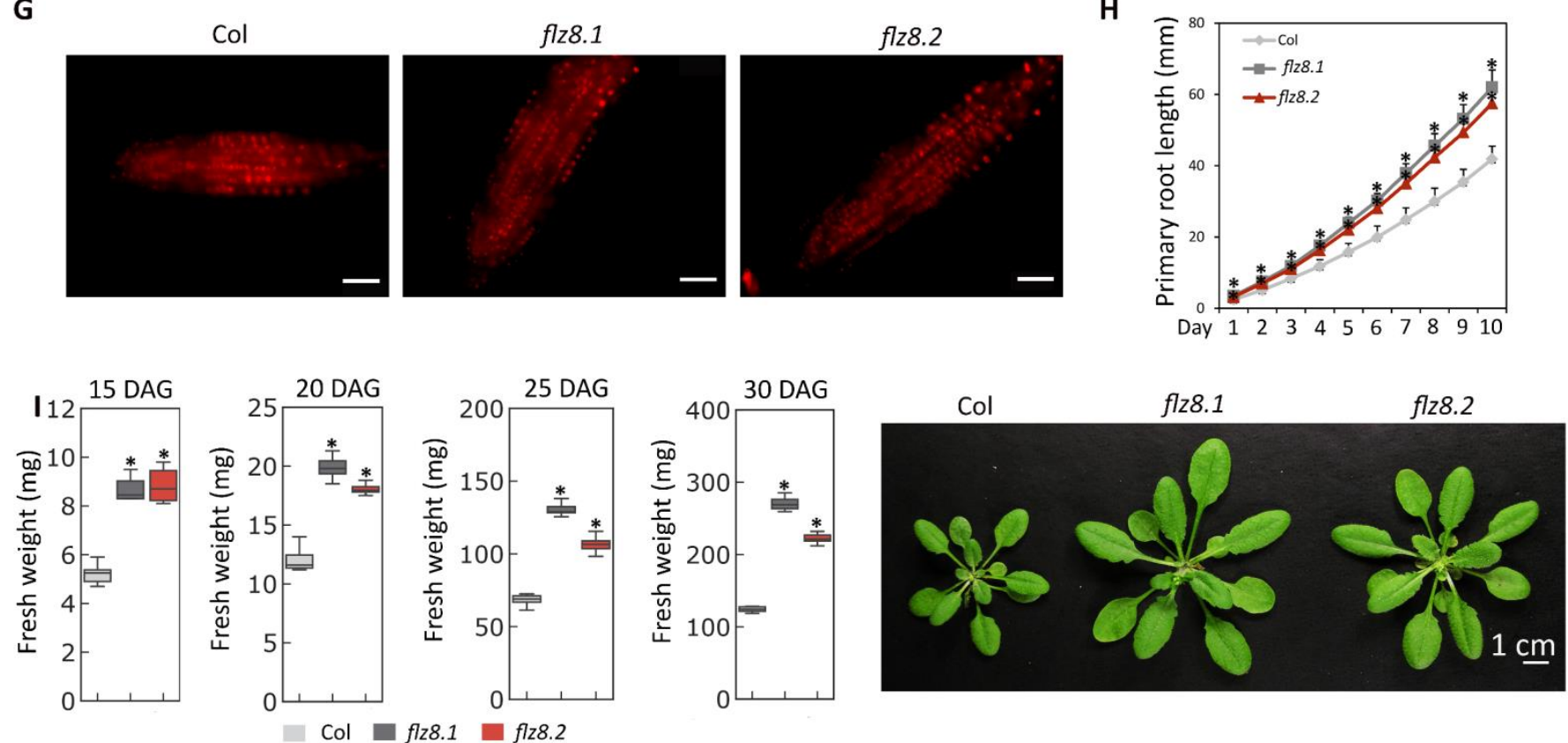

Fig. 3: Meristem activity and growth are enhanced in flz8 mutants.

(A) Gene structure of FLZ8 showing the T-DNA insertion site in flz8.1 and flz8.2 alleles. The position of primer sets (RT1 and RT2) used for qPCR is also shown in the gene structure. (B) Expression of $F L Z 8$ in mutant lines (one-way ANOVA, * $p \leq 0.05$, Bonferroni post-hoc test). (C) Cotyledon angle of WT and flz8 lines at 3 DAG. (D) and (E) Phenotype and measurement of biomass, primary root length, and lateral root number of WT and flz8 lines at 10 DAG. (F) Root meristem size (length and diameter) of WT and flz8 lines at 5 DAG. Blue arrow points to the quiescent centre and black arrow points to the junction between meristem and elongation zone (G) Edu (5-ethynyl-2'-deoxyuridine) staining showing root meristem activity of WT and flz8 lines at 5 DAG. (H) Root growth kinetics in WT and flz8 lines. (I) Measurement of rosette biomass at different rosette stages and phenotype of WT and flz8 lines at 30 DAG (one-way ANOVA, ${ }^{*} p \leq 0.05$, Bonferroni post-hoc test). 
A
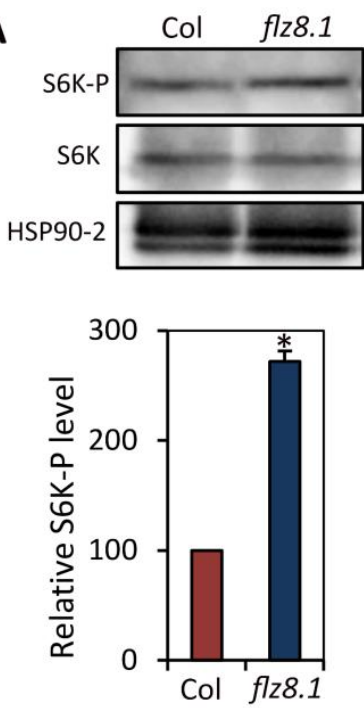
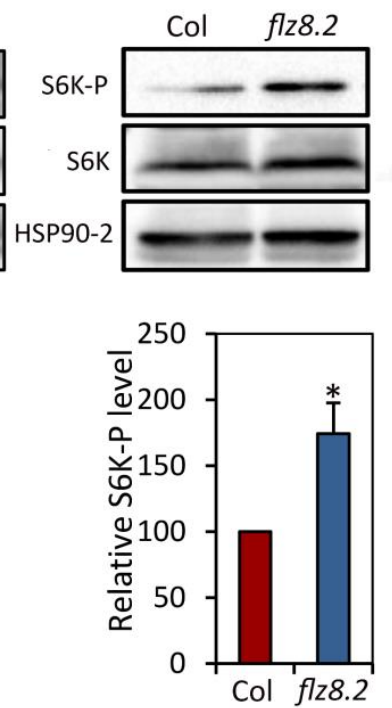

AZD8055
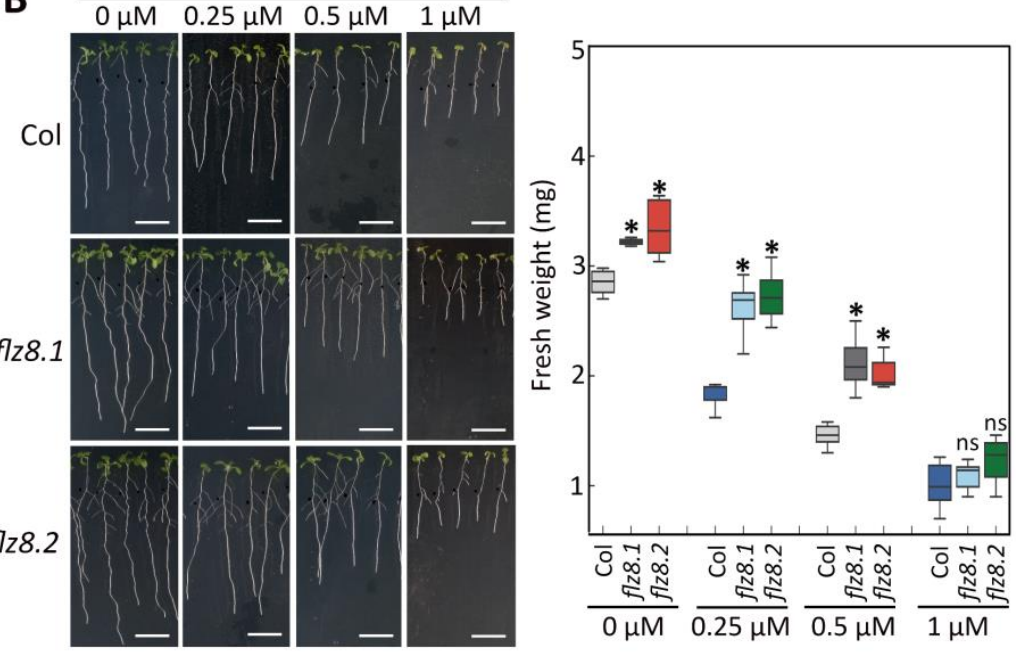

C

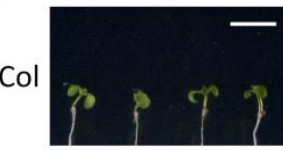

$f z 8.1$
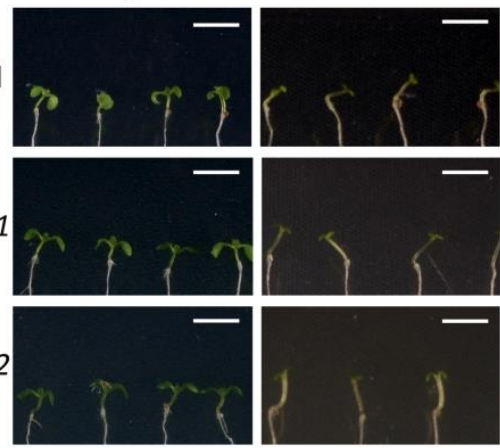

$30 \mathrm{mM}$ Suc
2 D Dark
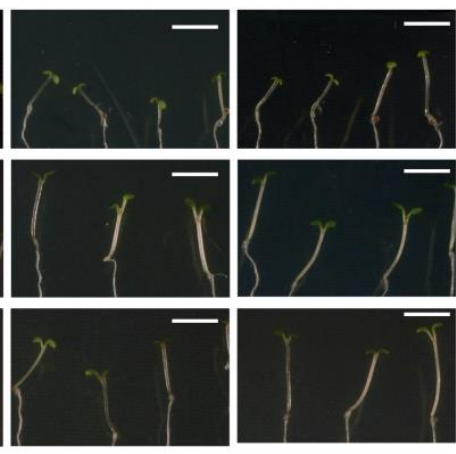

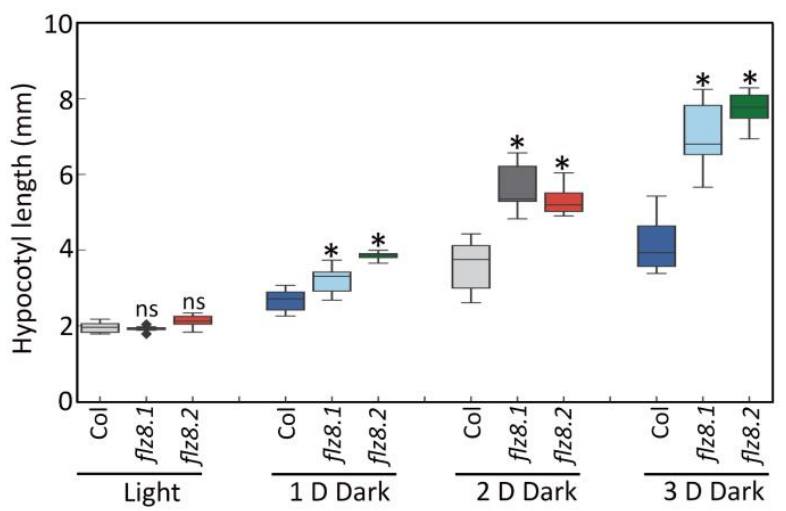

Fig. 4: TOR signaling is hyperactivated in flz8 mutants.

(A) Level of total and phosphorylated S6Ks (Thr-449 in S6K1 and Thr-455 in S6K2) in WT and flz8 mutants at 10 DAG. Relative level of S6K-P was quantified in comparison to total S6K (one-way ANOVA, * $p \leq 0.05$, Bonferroni post-hoc test). (B) Phenotype and fresh weight of WT and mutant lines in different concentrations of TOR inhibitor AZD8055 (two-way ANOVA, * $p \leq 0.05$, Bonferroni post-hoc test). (C) Sucrose-dependent hypocotyl elongation in WT and flz8.1 in response to dark treatment for 1,2 and 3 days (two-way ANOVA, * $p \leq 0.05$, Bonferroni post-hoc test). 
A
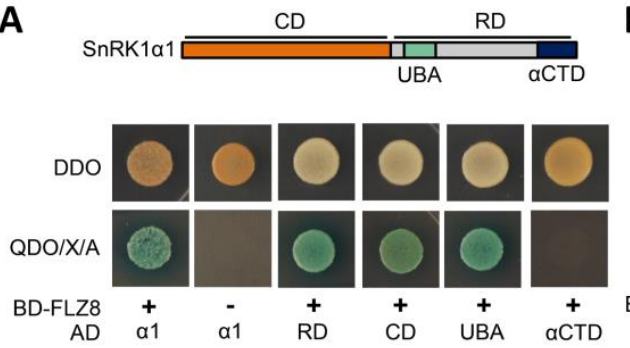

B

C

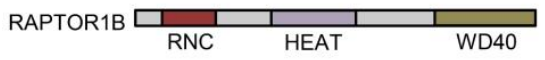

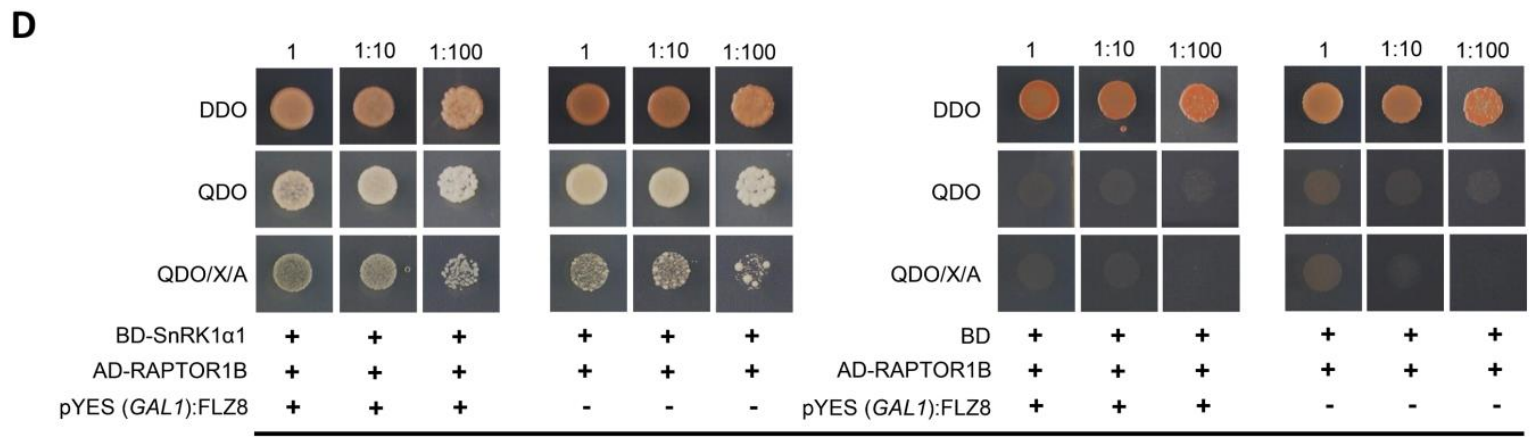

$\mathbf{E}$

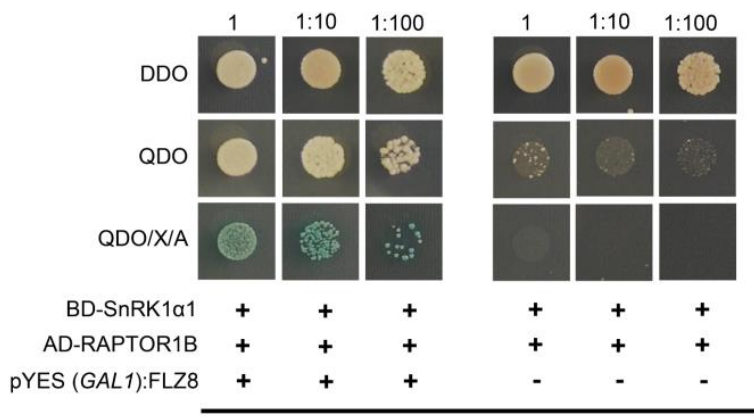

Glucose

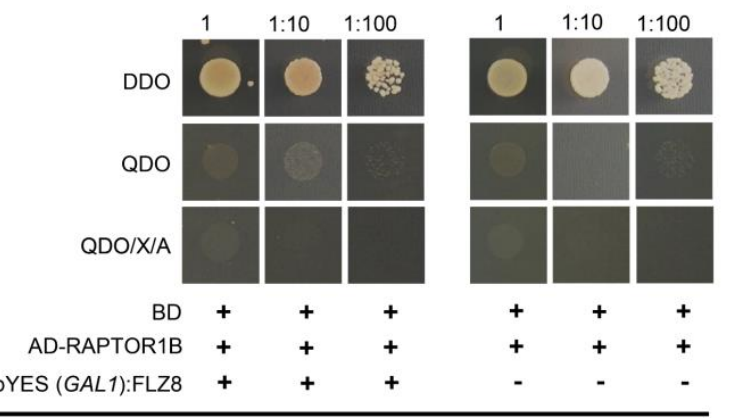

Galactose

Fig. 5: FLZ8 promotes the interaction of SnRK1a1 with RAPTOR1B

(A) Domain organization of SnRK1a1 and mapping of FLZ8-interaction sites in SnRK1a1 by Y2H analysis. (B) Interaction of FLZ8 with RAPTOR1B. (C) Domain organization of RAPTOR1B and mapping of FLZ8-interaction sites in RAPTOR1B. (D) and (E) Analysis of the interaction of SnRK1a1 and RAPTOR1B in the presence of FLZ8 by $\mathrm{Y} 3 \mathrm{H}$ analysis in glucose and galactose. For $\mathrm{Y} 2 \mathrm{H}$, the cotransformed yeast cells carrying different $A D$ and BD construct combinations were spotted on synthetic double dropout (DDO; -Trp/-Leu) and quadruple dropout medium supplemented with $40 \mu \mathrm{g} / \mathrm{mL}$ X-a-Gal and $200 \mathrm{ng} / \mathrm{mL}$ AbA (QDO/X/A; -Ade/-His/-Leu/-Trp/+ X$\alpha-\mathrm{Gal} /+\mathrm{AbA})$. For $\mathrm{Y} 3 \mathrm{H}$, the cotransformed yeast cells carrying different $A D$ and $\mathrm{BD}$ construct combinations along with pYES (GAL1) FLZ8) or pYES (GAL1) growing in glucose or galactose were spotted on DDO medium and weak (QDO) and strong (QDO/X/A) interaction screening medium. 
A
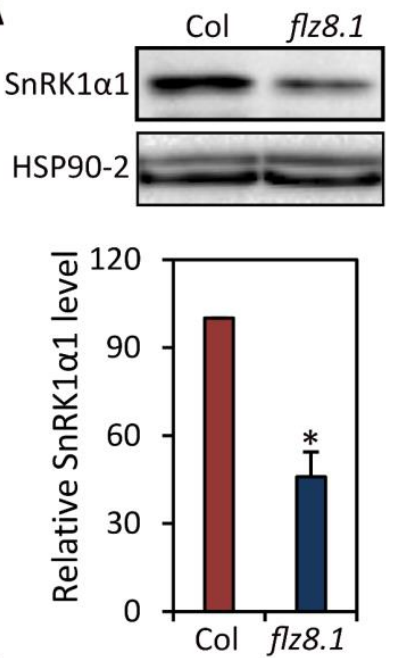

C

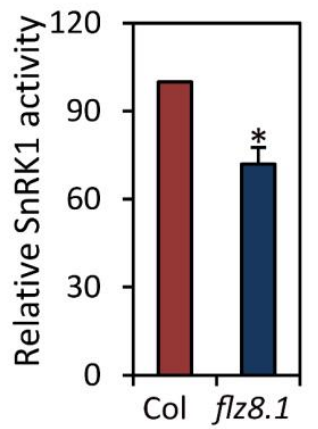

Col flz8.2
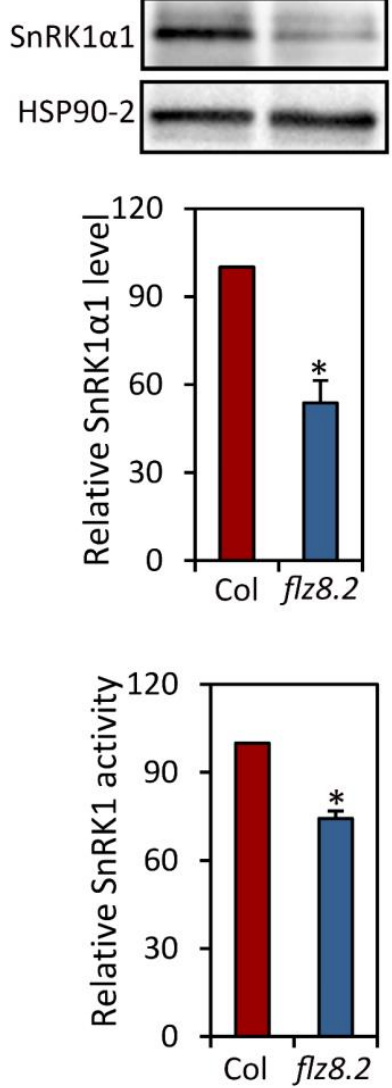

B
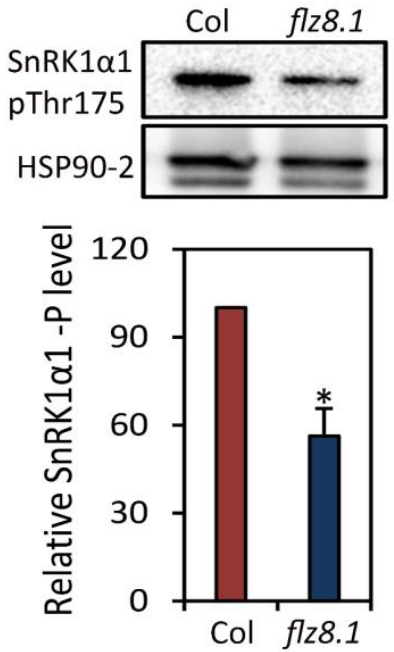

D

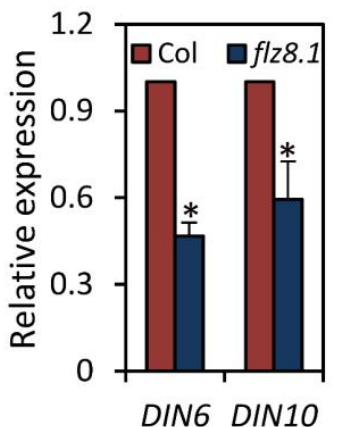

Col flz8.2
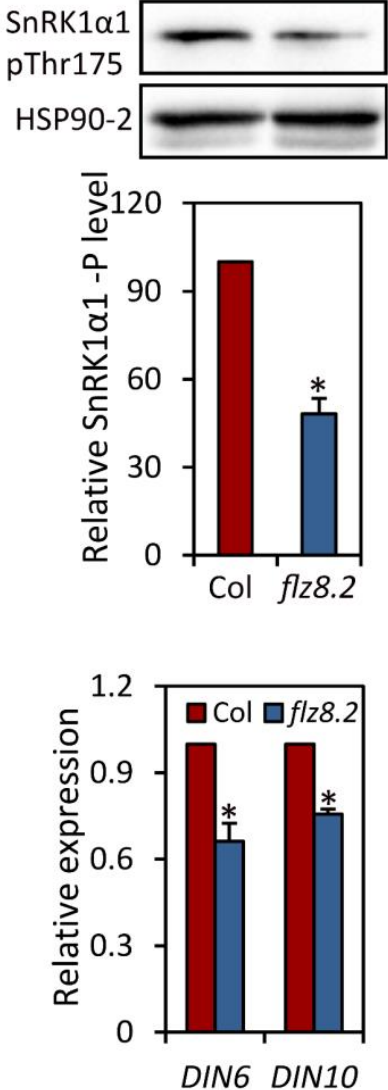

E

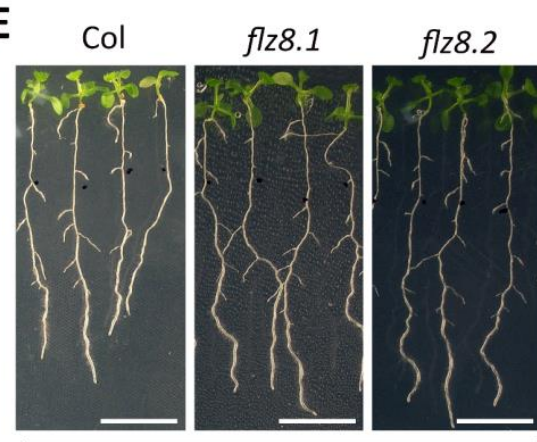

$0 \mu \mathrm{M}$ ABA

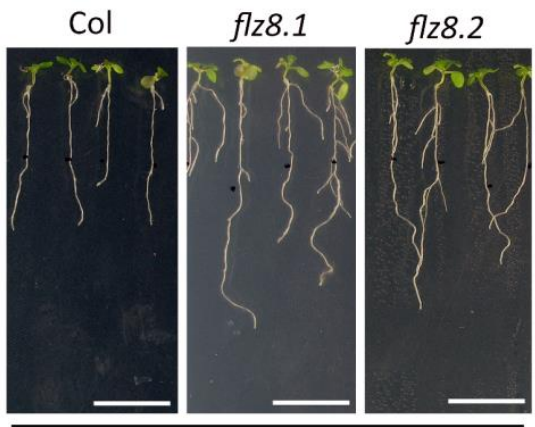

$5 \mu \mathrm{M}$ ABA

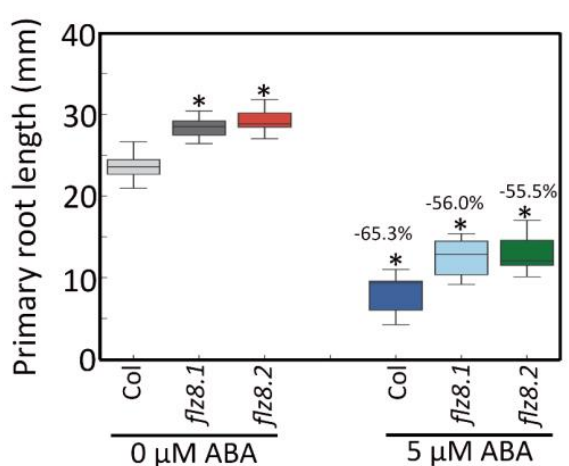

Fig. 6: SnRK1 signaling is attenuated in flz8 mutants.

(A) Level of SnRK1a1 in WT and flz8 mutants at 10 DAG. Relative level of SnRK1a1 was quantified in comparison to HSP90-2 level (one-way ANOVA, ${ }^{*} p \leq 0.05$, Bonferroni post-hoc test). (B) Level of phosphorylated SnRK1a1 (Thr-175) in WT and flz8 mutants at 10 DAG. Relative level of SnRK1a1-P was quantified in comparison to HSP90-2 level (oneway ANOVA, ${ }^{*} p \leq 0.05$, Bonferroni post-hoc test). (C) SnRK1 activity in 10 DAG flz8 mutants in comparison to WT in AMARA peptide assay (one-way ANOVA, ${ }^{*} p \leq 0.05$, Bonferroni post-hoc test). (D) Expression of SnRK1-activity reporter genes (DIN6 and DIN10) in 10 DAG flz8 mutants in comparison to WT (one-way ANOVA, ${ }^{*} p \leq 0.05$, Bonferroni post-hoc test). (E) Phenotype and primary root length of WT and flz 8 mutants in response to ABA treatment for 5 days (two-way ANOVA, ${ }^{*} \mathrm{p} \leq 0.05$, Bonferroni post-hoc test). 
